\title{
The unitary representations of the Poincaré group in any spacetime dimension
}

\author{
Xavier Bekaert $^{1 \star}$, Nicolas Boulanger ${ }^{2 \dagger}$
}

1 Institut Denis Poisson, Unité mixte de Recherche 7013, Université de Tours, Université d'Orléans, CNRS, Parc de Grandmont, 37200 Tours, France

2 Service de Physique de l'Univers, Champs et Gravitation, Université de Mons, UMONS Research Institute for Complex Systems, Place du Parc 20, 7000 Mons, Belgium

$\star$ xavier.bekaert@Impt.univ-tours.fr, $\uparrow$ nicolas.boulanger@umons.ac.be

\begin{abstract}
An extensive group-theoretical treatment of linear relativistic field equations on Minkowski spacetime of arbitrary dimension $D \geqslant 3$ is presented. An exhaustive treatment is performed of the two most important classes of unitary irreducible representations of the Poincaré group, corresponding to massive and massless fundamental particles. Covariant field equations are given for each unitary irreducible representation of the Poincaré group with non-negative mass-squared.
\end{abstract}

(c) (1) Copyright X. Bekaert and N. Boulanger. This work is licensed under the Creative Commons Attribution 4.0 International License.

Published by the SciPost Foundation.
Received 31-12-2020

Accepted 07-06-2021

Published 14-06-2021

Check for updates

doi:10.21468/SciPostPhysLectNotes.30

\section{Contents}

1 Group-theoretical preliminaries $\quad 2$

1.1 Universal covering of the Lorentz group 2

1.2 The Poincaré group and algebra 3

$\begin{array}{ll}1.3 \mathrm{ABC} \text { of unitary representations } & 4\end{array}$

2 Elementary particles as unitary irreducible representations of the isometry group 5

$\begin{array}{lll}3 & \text { Classification of the unitary representations } & 7\end{array}$

$\begin{array}{ll}3.1 \text { Induced representations } & 7\end{array}$

$\begin{array}{llr}3.2 & \text { Orbits and stability subgroups } & 8\end{array}$

$\begin{array}{ll}3.3 \text { Classification } & 10\end{array}$

4 Tensorial representations and Young diagrams $\quad 12$

$\begin{array}{lll}4.1 & \text { Symmetric group } & 12\end{array}$

$\begin{array}{lll}4.2 & \text { General linear group } & 15\end{array}$

$\begin{array}{ll}4.3 \text { Orthogonal group } & 18\end{array}$

4.4 Auxiliary variables $\quad 21$

$\begin{array}{ll}4.5 & \text { Euclidean group } \\ \end{array}$

5 Relativistic field equations $\quad 26$ 
$\begin{array}{lll}5.1 & \text { General procedure } & 27\end{array}$

$\begin{array}{lll}5.2 & \text { Massive representations } & 28\end{array}$

$\begin{array}{lll}5.3 & \text { Massless representations } & 30\end{array}$

$\begin{array}{lll}\text { 5.3.1 Helicity representations } & 30\end{array}$

5.3.2 Infinite spin representations $\quad 35$

$\begin{array}{lll}5.4 & \text { Tachyonic representations } & 36\end{array}$

$\begin{array}{lr}\text { A Siegel-Zwiebach equations } & 38\end{array}$

B Bargmann -Wigner programme in three dimensions $\quad 40$

B.1 Zero momentum representations 41

B.2 Massive representations 41

$\begin{array}{lll}\text { B.2.1 (Half-)integer spins } & 41\end{array}$

B.2.2 Fractional spins 41

$\begin{array}{lll}\text { B.3 Massless representations } & 42\end{array}$

$\begin{array}{lll}\text { B.3.1 Helicity representations } & 43\end{array}$

B.3.2 Infinite spin representations 43

B.4 Tachyonic representations 44

References $\quad 44$

\section{Group-theoretical preliminaries}

Elementary knowledge of the theory of Lie groups and their representations is assumed (see e.g. the textbooks $[1,2]$ or the lecture notes [3]). The basic definitions of the Lorentz and Poincaré groups together with some general facts on the theory of unitary representations are reviewed in order to fix the notation and settle down the prerequisites.

\subsection{Universal covering of the Lorentz group}

The group of linear homogeneous transformations $x^{\prime \mu}=\Lambda_{\nu}^{\mu} x^{v}(\mu, v=0,1, \ldots, D-1)$ preserving the Minkowski metric $\eta_{\mu \nu}$ of "mostly plus" signature $(-,+, \ldots,+)$,

$$
\Lambda^{T} \eta \Lambda=\eta,
$$

where $\Lambda^{T}$ denotes the matrix transpose of $\Lambda$, is called the Lorentz group $O(D-1,1)$.

A massless particle propagates on the light-cone $x^{2}=0$. Without loss of generality, one may consider that its momentum points along the $(D-1)$ th spatial direction. Then it turns out to be convenient to make use of the light-cone coordinates

$$
x^{ \pm}=\frac{1}{\sqrt{2}}\left(x^{D-1} \pm x^{0}\right) \text { and } x^{m} \quad(m=1, \ldots, D-2),
$$

where the Minkowski metric reads $\eta_{++}=0=\eta_{--}, \eta_{+-}=1=\eta_{-+}$and $\eta_{m n}=\delta_{m n}$ $(m, n=1, \ldots, D-2)$.

On physical grounds, one will mainly be interested in the matrices $\Lambda$ 's with determinant +1 and such that $\Lambda_{0}^{0} \geqslant 0$. It can be shown that such matrices $\Lambda$ 's also form a group that one calls the proper orthochronous Lorentz group denoted by $S O(D-1,1)^{\uparrow}$. It is connected to the identity, but not simply connected, that is to say, there exist loops in the group manifold 
$S O(D-1,1)^{\uparrow}$ which are not continuously contractible to a point. In order to study the representations (reps) of $S O(D-1,1)^{\uparrow}$, one may first determine its universal covering group, i.e. the Lie group which is simply connected and whose Lie algebra is isomorphic to $\mathfrak{s o}(D-1,1)$, the Lie algebra of $S O(D-1,1)^{\uparrow}$. For $D \geqslant 4$, the universal covering group, denoted $\operatorname{Spin}(D-1,1)$, is the double cover of $S O(D-1,1)^{\uparrow}$. The spin groups $S \operatorname{pin}(D-1,1)$ have no intrinsically projective representations. Therefore, a single (or double) valued "representation" of $S O(D-1,1)^{\uparrow}$ is meant to be a genuine representation of $\operatorname{Spin}(D-1,1)$.

Warning: The double cover of $S O(2,1)^{\uparrow}$ is the group $S U(1,1)$, in close analogy to the fact that the double cover of $S O(3)$ is $S U(2)$. The group $S U(2)$ is also the universal covering group of $S O(3)$, but beware that the universal cover of $S O(2,1)^{\uparrow}$ is actually $\mathbb{R}^{3}$, covering $S O(2,1)^{\uparrow}$ infinitely many times. Thus one may not speak of the spin group for the case of the proper orthochronous Lorentz group in spacetime dimension three. The analogue is that the universal cover of $S O(2) \cong U(1)$ is $\mathbb{R}$, that covers $U(1)$ infinitely many times, so that one may not speak of the spin group for the degenerate case of the rotation group in two spatial dimensions.

\subsection{The Poincaré group and algebra}

The transformations

$$
x^{\prime \mu}=\Lambda^{\mu}{ }_{v} x^{\mu}+a^{\mu},
$$

where $a$ is a spacetime translation vector, form the group of all inhomogeneous Lorentz transformations. If one denotes a general transformation by $(\Lambda, a)$, the multiplication law in the Poincaré group is given by

$$
\left(\Lambda_{2}, a_{2}\right) \cdot\left(\Lambda_{1}, a_{1}\right)=\left(\Lambda_{2} \Lambda_{1}, a_{2}+\Lambda_{2} a_{1}\right),
$$

so that the inhomogeneous Lorentz group is the semi-direct product denoted by

$$
I O(D-1,1)=\mathbb{R}^{D} \rtimes O(D-1,1) .
$$

The subgroup ISO $(D-1,1)^{\uparrow}$ of inhomogeneous proper orthochronous Lorentz transformations is called the Poincaré group. The Lie algebra iso $(D-1,1)$ of the Poincaré group is presented by the generators $\left\{P_{\mu}, M_{\nu \rho}\right\}$ and by the commutation relations

$$
\begin{aligned}
i\left[M_{\mu \nu}, M_{\rho \sigma}\right] & =\eta_{\nu \rho} M_{\mu \sigma}-\eta_{\mu \rho} M_{\nu \sigma}-\eta_{\sigma \mu} M_{\rho v}+\eta_{\sigma v} M_{\rho \mu} \\
i\left[P_{\mu}, M_{\rho \sigma}\right] & =\eta_{\mu \rho} P_{\sigma}-\eta_{\mu \sigma} P_{\rho}, \\
i\left[P_{\mu}, P_{\rho}\right] & =0 .
\end{aligned}
$$

Two subalgebras must be distinguished: the Lie algebra $\mathfrak{s o}(D-1,1)$ of the Lorentz group presented by the generators $\left\{M_{v \rho}\right\}$ and by the commutation relations (1), and the Lie algebra $\mathbb{R}^{D}$ of the Abelian translation group presented by the generators $\left\{P_{\mu}\right\}$ and by the commutation relations (3). The latter algebra $\mathbb{R}^{D}$ is an ideal of the Poincaré algebra, as can be seen from (2). Altogether, this implies that the Lie algebra of the Poincaré group is the semi-direct sum $\mathfrak{i s o}(D-1,1)=\mathbb{R}^{D} \boxplus \mathfrak{s o}(D-1,1)$.

The Casimir elements of a Lie algebra $\mathfrak{g}$ are homogeneous polynomials in the generators of $\mathfrak{g}$ providing a distinguished basis of the center $\mathcal{Z}(\mathcal{U}(\mathfrak{g}))$ of the universal enveloping algebra $\mathcal{U}(\mathfrak{g})$ (see e.g. the part $\mathrm{V}$ of the lecture notes [3]). The quadratic Casimir operator of the Lorentz algebra $\mathfrak{s o}(D-1,1)$ is the square of the generators $M_{\mu \nu}$ :

$$
\mathcal{C}_{2}(\mathfrak{s o}(D-1,1))=\frac{1}{2} M^{\mu v} M_{\mu v}
$$


The quadratic Casimir operator of the Poincaré algebra iso $(D-1,1)$ is the square of the momentum

$$
\mathcal{C}_{2}(\mathfrak{i s o}(D-1,1))=-P^{\mu} P_{\mu},
$$

while the quartic Casimir operator is

$$
\mathcal{C}_{4}(\mathfrak{i s o}(D-1,1))=-\frac{1}{2} P^{2} M_{\mu \nu} M^{\mu v}+M_{\mu \rho} P^{\rho} M^{\mu \sigma} P_{\sigma},
$$

which, for $D=4$, is the square of the Pauli-Lubanski vector $W^{\mu}$,

$$
W^{\mu}:=\frac{1}{2} \varepsilon^{\mu \nu \rho \sigma} M_{\nu \rho} P_{\sigma} .
$$

\section{3 $\mathrm{ABC}$ of unitary representations}

The mathematical property that all non-trivial unitary reps of a non-compact simple Lie group must be infinite-dimensional has some physical significance, as will be reviewed later.

Finite-dimensional unitary reps of non-compact simple Lie groups: Let $\mathrm{U}: G \rightarrow U(n)$ be a unitary representation of a Lie group $G$ acting on a (real or complex) Hilbert space $\mathcal{H}$ of finite dimension $n \in \mathbb{N}$. Then $\mathrm{U}$ is completely reducible. Moreover, if $\mathrm{U}$ is irreducible and if $G$ is a connected simple non-compact Lie group, then $\mathrm{U}$ is the trivial representation.

Proof: For the property that $\mathrm{U}$ is completely reducible, we refer e.g. to the proof of the proposition 5.15 in [1]. The image $\mathrm{U}(G)$ for any unitary representation $\mathrm{U}$ defines a subgroup of $U(n)$. Moreover, the kernel of $U$ is a normal subgroup of the simple group $G$. Therefore, either the representation is trivial and $\operatorname{ker} U=G$, or it is faithfull and $\operatorname{ker} U=\{e\}$. In the latter case, $U$ is invertible and its image is isomorphic to its domain, $\mathrm{U}(G) \cong G$. Actually, the image $\mathrm{U}(G)$ is a non-compact subgroup of $U(n)$ because if $\mathrm{U}(G)$ was compact, then $\mathrm{U}^{-1}(\mathrm{U}(G))=G$ would be compact since $\mathrm{U}^{-1}$ is a continuous map. But the group $U(n)$ is compact, thus it cannot contain a non-compact subgroup. Therefore the representation cannot be faithful, so that it is trivial. (A different proof of the second part of the theorem may be found in the section 8.1.B of [2].)

Another mathematical result which is of physical significance is the following theorem on unitary irreducible representations (UIRs) of compact Lie groups.

Unitary reps of compact Lie groups: Let $\mathrm{U}$ be a UIR of a compact Lie group G, acting on a (real or complex) Hilbert space $\mathcal{H}$. Then $\mathcal{H}$ is finite-dimensional. Moreover, every unitary representation of $G$ is a direct sum of UIRs (the sum may be infinite).

Proof: The proofs are somewhat lengthy and technical so we refer to the section 7.1 of [2] for complete details.

\section{Examples of (not so) simple groups:}

- On the one hand, all (pseudo)-orthogonal groups $S O(p, q)$ are either Abelian $(p+q=2)$, non-simple $(p+q=4)$ or simple $(p+q=3$ and $p+q>4)$. Moreover, the orthogonal groups $(p q=0)$ are compact, while the pseudo-orthogonal groups $(p q \neq 0)$ are non-compact.

- On the other hand, the inhomogeneous Lorentz group $I O(D-1,1)$ is non-compact (both $\mathbb{R}^{D}$ and $O(D-1,1)$ are non-compact) but it is not semi-simple (because its normal subgroup $\mathbb{R}^{D}$ is Abelian). 


\section{Elementary particles as unitary irreducible representations of the isometry group}

Except for the final remarks, this section is based almost ad verbatim on the introduction of the illuminating work of Bargmann and Wigner [4], modulo some changes of notation and terminology in order to follow the modern conventions.

The wave functions $|\psi\rangle$ describing the possible states of a quantum-mechanical system form a linear vector space $\mathcal{H}$ which, in general, is infinite-dimensional and on which a positivedefinite inner product $\langle\phi \mid \psi\rangle$ is defined for any two wave functions $|\phi\rangle$ and $|\psi\rangle$ (i.e. they form a Hilbert space). The inner product usually involves an integration over the whole configuration or momentum space and, for particles of non-vanishing spin, a summation over the spin indices.

If the wave functions in question refer to a free particle and satisfy relativistic wave equations, there exists a correspondence between the wave functions describing the same state in different Lorentz frames. The transformations considered here form the group of all inhomogeneous Lorentz transformations (including translations of the origin in space and time). Let $|\psi\rangle$ and $|\psi\rangle^{\prime}$ be the wave functions of the same state in two Lorentz frames $L$ and $L^{\prime}$, respectively. Then $|\psi\rangle^{\prime}=U(\Lambda, a)|\psi\rangle$, where $U(\Lambda, a)$ is a linear unitary operator which depends on the transformation $(\Lambda, a)$ leading from $L$ to $L^{\prime}$. By a proper normalization, $U$ is determined by $\Lambda$ up to a factor \pm 1 . Moreover, the operators $U$ form a single- or double-valued representation of the inhomogeneous Lorentz group, i.e., for a succession of two transformations $\left(\Lambda_{1}, a_{1}\right)$ and $\left(\Lambda_{2}, a_{2}\right)$, we have

$$
U\left(\Lambda_{2}, a_{2}\right) U\left(\Lambda_{1}, a_{1}\right)= \pm U\left(\Lambda_{2} \Lambda_{1}, a_{2}+\Lambda_{2} a_{1}\right) .
$$

Since all Lorentz frames are equivalent for the description of our system, it follows that, together with $|\psi\rangle, U(\Lambda, a)|\psi\rangle$ is also a possible state viewed from the original Lorentz frame $L$. Thus, the vector space $\mathcal{H}$ contains, with every $|\psi\rangle$, all transforms $U(\Lambda, a)|\psi\rangle$, where $(\Lambda, a)$ is any inhomogenous Lorentz transformation.

The operators $U$ may also replace the wave equation of the system. In our discussion, we use the wave functions in the "Heisenberg" representation, so that a given $|\psi\rangle$ represents the system for all times, and may be chosen as the "Schrödinger" wave function at time $t=0$ in a given Lorentz frame $L$. To find $|\psi\rangle_{t_{0}}$, the Schrödinger function at time $t_{0}$, one must therefore transform to a frame $L^{\prime}$ for which $t^{\prime}=t-t_{0}$, while all other coordinates remain unchanged. Then $|\psi\rangle_{t_{0}}=U(\Lambda, a)|\psi\rangle$, where $(\Lambda, a)$ is the transformation leading from $L$ to $L^{\prime}$.

A classification of all unitary representations of the inhomogeneous Lorentz group, i.e. of all solution of (7), amounts, therefore, to a classification of all possible relativistic wave equations. Two reps $U$ and $\widetilde{U}=V U V^{-1}$, where $V$ is a fixed unitary operator, are equivalent. If the system is described by wave functions $|\psi\rangle$, the description by

$$
\widetilde{|\psi\rangle}=V|\psi\rangle
$$

is isomorphic with respect to linear superposition, with respect to forming the inner product of two wave functions, and also with respect to the transition from one Lorentz frame to another. In fact, if $|\psi\rangle^{\prime}=U(\Lambda, a)|\psi\rangle$, then

$$
{\widetilde{|\psi\rangle^{\prime}}}^{\prime}=V|\psi\rangle^{\prime}=V U(\Lambda, a) V^{-1} \widetilde{|\psi\rangle}=\widetilde{U}(\Lambda, a) \widetilde{|\psi\rangle} .
$$

Thus, one obtains classes of equivalent wave equations. Finally, it is sufficient to determine the irreducible representations (irreps) since any other may be built from them.

Two descriptions which are equivalent according to (8) may be quite different in appearance. The best known example is the description of the electromagnetic field by the field 
strength and the vector potential, respectively. It cannot be claimed either that equivalence in the sense of (8) implies equivalence in every physical aspect. It should be emphasized that any selection of one among the equivalent systems involves an explicit or implicit assumption as to possible interactions, etc. Our analysis is necessarily restricted to free particles and does not lead to any assertion about possible interactions.

The present discussion is not based on any hypothesis about the structure of the wave equations provided that they be covariant. In particular, it is not necessary to assume differential equations in configuration space. But it is a result of the group-theoretical analysis that every irreducible field equation is equivalent, in the sense of (8), to a system of differential equations for fields on Minkowski spacetime.

\section{Remarks:}

- An important theorem proved by Wigner is that any symmetry transformation that is continuously related to the identity must be represented by a linear unitary operator (see e.g. the appendix A of [5]). Strictly speaking, physical states are represented by rays in a Hilbert space. Therefore the unitary representations of the symmetry group are actually understood to be projective representations. In spacetime dimensions $D \geqslant 4$, this subtlety ${ }^{1}$ reduces to the standard distinction between single and double valued representations of the Poincaré group, as was taken for granted in the text.

- Notice that the previous discussion remains entirely valid if the Minkowski spacetime $\mathbb{R}^{D-1,1}$ is replaced everywhere by any other maximally symmetric spacetime (i.e. de Sitter spacetime $d S_{D}$, or anti de Sitter spacetime $A d S_{D}$ ) under the condition that the inhomogeneous Lorentz group $I O(D-1,1)$ be also replaced everywhere by the corresponding group of isometries (respectively, $O(D, 1)$ or, $O(D-1,2)$ ).

- In first-quantization, particles are described by fields on the spacetime and isometries are represented by unitary operators. A particle is said to be "elementary" if the representation is irreducible, and "composite" if the representation is made of a product of irreps.

- A modern point of view on Quantum Field Theory [5] is that a quantum field (not to be confused with the state vector discussed above) is an operator defined at each point of space and time, that acts in a Fock space of states, the field being represented by a superposition, for different values of the momentum, of one-particule annihilation and creation operators for particle and the associated antiparticle. The approach of [5] is to build up the quantum field by imposing Lorentz invariance at every stage. To quote Weinberg, the field equation satisfied by the quantum field arises almost incidentally, as a byproduct of his construction.

- A unitary representation of the isometry group describes the one-particle Hilbert space of states. The group-theoretical argument of Bargmann and Wigner [4] applies to the oneparticule states of a free particle. ${ }^{2}$ The classification of the UIRs of the Poincaré group indeed yields the Klein-Gordon equation for a massive particle, or the D'Alembert equation in the case of a massless particle [4]. This comes automatically from the group-theoretical analysis and is not an assumption.

Summary: On the one hand, the rules of quantum mechanics imply that quantum symmetries correspond to unitary representations of the symmetry group carried by the Hilbert space of physical states. Furthermore, if time translations constitute a one-parameter subgroup of the symmetry group, then the Schrödinger equation for the time evolution of a state vector essentially is a unitary representation of this subgroup. On the other hand, the principle of relativity dictates that the isometries of spacetime be symmetries of the physical system. All together, this implies that linear relativistic field equations may be identified with unitary reps of the isometry group.

\footnotetext{
${ }^{1}$ The case $D=3$ is even more subtle and is treated in Appendix B.

${ }^{2}$ See e.g. Eq. (2.5.1) of [5] where the one-particle state vectors are denoted by $\Psi_{p, \sigma}$.
} 


\section{Classification of the unitary representations}

\subsection{Induced representations}

The method of induced reps was introduced by Wigner in his seminal paper [6] on the unitary representations of the inhomogeneous Lorentz group $I O(3,1)$ in four spacetime dimensions, which admits a straightforward generalization to any spacetime dimension $D$, as reviewed now. The content of this subsection finds its origin in the section 2.5 of the comprehensive textbook [5].

From (3) one sees that all the translation generators commute with each other, so it is natural to express physical states $|\psi\rangle$ in terms of eigenvectors of the translation generators $P^{\mu}$. Introducing a label $\sigma$ to denote all other degrees of freedom, one thus considers states $\Psi_{q, \sigma}$ with $P_{\mu} \Psi_{q, \sigma}=q_{\mu} \Psi_{q, \sigma}$. From the infinitesimal translation $U=\mathbb{1}-i P^{\mu} \epsilon_{\mu}$ and repeated applications of it, one finds that finite translations are represented on $\mathcal{H}$ by $U(\mathbb{1}, a)=\exp \left(-i P^{\mu} a_{\mu}\right)$, so one has

$$
U(\mathbb{1}, a) \Psi_{q, \sigma}=e^{-i q \cdot a} \Psi_{q, \sigma} .
$$

Using (2), one sees that the effect of operating on $\Psi_{p, \sigma}$ with a quantum homogeneous transformation $U(\Lambda, 0) \equiv U(\Lambda)$ is to produce an eigenvector of the translation generators with eigenvalue $\Lambda p$ :

$$
\begin{aligned}
P^{\mu} U(\Lambda) \Psi_{p, \sigma} & =U(\Lambda)\left[U^{-1}(\Lambda) P^{\mu} U(\Lambda)\right] \Psi_{p, \sigma}=U(\Lambda)\left(\left(\Lambda^{-1}\right)_{\rho}{ }^{\mu} P^{\rho}\right) \Psi_{p, \sigma} \\
& =\Lambda_{\rho}^{\mu} p^{\rho} U(\Lambda) \Psi_{p, \sigma},
\end{aligned}
$$

since $\left(\Lambda^{-1}\right)_{\rho}{ }^{\mu}=\Lambda_{\rho}^{\mu}$. Hence $U(\Lambda) \Psi_{p, \sigma}$ must be a linear combination of the states $\Psi_{\Lambda p, \sigma}$ :

$$
U(\Lambda) \Psi_{p, \sigma}=\sum_{\sigma^{\prime}} C_{\sigma^{\prime} \sigma}(\Lambda, p) \Psi_{\Lambda p, \sigma^{\prime}} .
$$

In general, it is possible by using suitable linear combinations of the $\Psi_{p, \sigma}$ to choose the $\sigma$ labels in such a way that the matrix $C_{\sigma^{\prime} \sigma}(\Lambda, p)$ is block-diagonal; in other words, so that the $\Psi_{p, \sigma}$ with $\sigma$ within any one block by themselves furnish a representation of the Poincaré group. It is natural to identify the states of a specific particle type with the components of a representation of the Poincaré group which is irreducible, in the sense that it cannot be further decomposed in this way. It is clear from (9) that all states $\Psi_{p, \sigma}$ in an irrep of the Poincaré group have momenta $p^{\mu}$ belonging to the orbit of a single fixed momentum, say $q^{\mu}$.

One has to work out the structure of the coefficients $C_{\sigma^{\prime} \sigma}(\Lambda, p)$ in irreducible representations of the Poincaré group. In order to do that, note that the only functions of $p^{\mu}$ that are left invariant by all transformations $\Lambda^{\mu}{ }_{v} \in S O(D-1,1)^{\uparrow}$ are, of course, $p^{2}=\eta_{\mu \nu} p^{\mu} p^{v}$ and, for $p^{2} \leqslant 0$, also the sign of $p^{0}$. Hence, for each value of $p^{2}$, and (for $p^{2} \leqslant 0$ ) each sign of $p^{0}$, one can choose a standard four-momentum, say $q^{\mu}$, and express any $p^{\mu}$ of this class as

$$
p^{\mu}=L_{v}^{\mu}(p) q^{v}
$$

where $L^{\mu}{ }_{v}$ is some standard proper orthochronous Lorentz transformation that depends on $p^{\mu}$, and also implicitly on our choice of $q^{\mu}$. One can define the states $\Psi_{p, \sigma}$ of momentum $p^{\mu}$ by

$$
\Psi_{p, \sigma} \equiv N(p) U(L(p)) \Psi_{q, \sigma},
$$

where $N(p)$ is a numerical normalization factor. Operating on (10) with an arbitrary homogeneous Lorentz transformation $U(\Lambda)$, one now finds

$$
\begin{aligned}
U(\Lambda) \Psi_{p, \sigma} & =N(p) U(\Lambda L(p)) \Psi_{q, \sigma} \\
& =N(p) U(L(\Lambda p)) U\left(L^{-1}(\Lambda p) \Lambda L(p)\right) \Psi_{q, \sigma} .
\end{aligned}
$$


The point of this last step is that the Lorentz transformation $L^{-1}(\Lambda p) \Lambda L(p)$ takes $q$ to $L(p) q=p$, then to $\Lambda p$, and finally back to $q$, so it belongs to the subgroup of the Lorentz group consisting of Lorentz transformations $W_{v}^{\mu}$ that leave $q^{\mu}$ invariant : $W_{\nu}^{\mu} q^{v}=q^{\mu}$. This stability subgroup is called the little group corresponding to $q$. For any $W, \bar{W}$ in the little group, one has

$$
U(W) \Psi_{q, \sigma}=\sum_{\sigma^{\prime}} D_{\sigma^{\prime} \sigma}^{q}(W) \Psi_{q, \sigma^{\prime}}
$$

and

$$
D_{\sigma^{\prime} \sigma}^{q}(\bar{W} W)=\sum_{\sigma^{\prime \prime}} D_{\sigma^{\prime} \sigma^{\prime \prime}}^{q}(\bar{W}) D_{\sigma^{\prime \prime} \sigma}^{q}(W)
$$

that is to say, the coefficients $D^{q}(W)$ furnish a representation of the little group. In particular, for $W(\Lambda, p) \equiv L^{-1}(\Lambda p) \Lambda L(p)$, the equation (11) becomes

$$
U(\Lambda) \Psi_{p, \sigma}=N(p) \sum_{\sigma^{\prime}} D_{\sigma^{\prime} \sigma}(W(\Lambda, p)) U(L(\Lambda p)) \Psi_{q, \sigma^{\prime}}
$$

or, recalling the definition (10),

$$
U(\Lambda) \Psi_{p, \sigma}=\frac{N(p)}{N(\Lambda p)} \sum_{\sigma^{\prime}} D_{\sigma^{\prime} \sigma}(W(\Lambda, p)) \Psi_{\Lambda p, \sigma^{\prime}} .
$$

Apart from the question of normalization, the problem of determining the coefficients $C_{\sigma^{\prime} \sigma}$ in the transformation rule (9) has been reduced to the problem of determining the coefficients $D_{\sigma^{\prime} \sigma}$. In other words, the problem of determining all possible irreps of the Poincaré group has been reduced to the problem of finding all possible irreps of the little group, depending on the class of momentum to which $q^{\mu}$ belongs. This approach, of deriving representations of a semi-direct product like the inhomogeneous Lorentz group from the representations of the stability subgroup, is called the method of induced representations.

The wave function $\Psi_{p, \sigma}$ depends on the momentum, therefore its Fourier transform $\Psi_{x, \sigma}$ depends on the spacetime coordinate, so that the wave function is called a (complex) field on Minkowski spacetime $\mathbb{R}^{D-1,1}$ and the quantities $\Psi_{x, \sigma}$ at fixed $x$ and for varying $\sigma$ are referred to as its physical components at $x$.

\subsection{Orbits and stability subgroups}

The orbit of a given non-vanishing vector $q^{\mu}$ of Minkowski spacetime $\mathbb{R}^{D-1,1}$ under Lorentz transformations is, by definition, the hypersurface of constant momentum square $p^{2}$. Geometrically speaking, it is a quadric of curvature radius $m>0$. More precisely, the hypersurface

- $p^{2}=-m^{2}$ is a two-sheeted hyperboloid, each sheet of which is called a mass-shell. The corresponding UIR is said to be massive.

- $p^{2}=0$ is a cone, each half of which is called a light-cone. The corresponding UIR is said to be massless $(m=0)$.

- $p^{2}=+m^{2}$ is a one-sheeted hyperboloid. The corresponding UIR is said to be tachyonic.

Orthochronous Lorentz transformations preserve the sign of the time component of vectors of non-positive momentum-squared, thus the orbit of a time-like (light-like) vector is the massshell (respectively, light-cone) to which the corresponding vector belongs.

\section{Remarks:}

- Notice that the Hilbert space carrying the irrep is indeed an eigenspace of the quadratic Casimir operator (5), the eigenvalue of which is $\mathcal{C}_{2}= \pm m^{2}$ (the eigenvalue is real because the 
representation is unitary), as it should according to Schur's lemma. Moreover, the quadratic Casimir classifies the UIRs but does not entirely characterize them.

- For any pair of fields that transform in the same UIR of the Poincaré group, the Poincaréinvariant scalar product for $p^{2} \leqslant 0$ is given by $\langle\Psi \mid \Phi\rangle=\int d^{D} p \delta\left(p^{2}+m^{2}\right) \Theta\left(p^{0}\right) \sum_{\sigma} \Psi_{p, \sigma}^{*} \Phi_{p, \sigma}$. - As quoted in Section 2, it is not necessary to assume differential equations in position space, because the group-theoretical analysis directly leads to a wave function which is a function of the momenta on the orbit, the Fourier transform of which is a function in position space obeying the Klein-Gordon equation $\square \Psi_{x, \sigma}= \pm m^{2} \Psi_{x, \sigma}$. By a slight abuse of terminology, states or fields that satisfy their relativistic equations of motion are called "on-(mass-)shell" in physics literature, while those for which those equations have not been imposed are called "off-shell".

By going to a rest-frame, it is easy to show that the stabilizer of a time-like vector $q^{\mu}=$ $(m, \overrightarrow{0}) \neq 0$ is the rotation subgroup $S O(D-1) \subset S O(D-1,1)^{\uparrow}$. For a space-like vector, one may choose a frame such that the non-vanishing momentum is along the $(D-1)$ th spatial axis: $q^{\mu}=(0,0, \ldots, 0, m) \neq 0$. Thus its stabilizer is the subgroup $S O(D-2,1)^{\uparrow} \subset S O(D-1,1)^{\uparrow}$. In the case of a light-like vector, the little group "is not quite so obvious" to determine, as was stressed by Wigner himself [7]. It clearly contains the rotation group $S O(D-2)$ in the spacelike hyperplane $\mathbb{R}^{D-2}$ transverse to the light-ray along the momentum. Now, we will provide an algebraic proof that the stabilizer of a light-like vector is the Euclidean group $I S O(D-2)$. According to Wigner, reviewing his $D=4$ analysis, "no simple argument is known (...) to show directly that the group of Lorentz transformations which leave a null vector invariant is isomorphic to the two-dimensional Euclidean group, desirable as it would be to have such an argument. Clearly, there is no plane in the four-space of momenta in which these transformations could be interpreted directly as displacements (...) because all transformations considered here are homogeneous" [7]. Even though there is no simple geometric way to understand this fact, the algebraic proof reviewed here is rather straightforward.

Proof: By going in a light-cone frame (see Section 1.1), it is possible to express the components of a momentum $p^{\mu}$ obeying $p^{2}=0$ as $p_{\mu}=\left(p_{-}, 0,0, \ldots, 0\right)$. In words, one can set the component $p_{+}$to zero, as well as all the transverse components $p_{m}(m=1, \ldots, D-2)$. The condition that the component $p_{-}$be unaffected by a Lorentz transformation translates as $0 \stackrel{!}{=} i\left[p_{-}, M_{\nu \rho}\right]=\eta_{-v} p_{\rho}-\eta_{-\rho} p_{v}$ due to (2). Obviously, the transformation generated by $M_{+-}$does modify $p_{-}$, hence it cannot be part of the little group for $p$. The other Lorentz generators preserve $p_{-}$, but they should also satisfy the equations $\left[p_{m}, M_{\mu \nu}\right]=0=\left[p_{+}, M_{\mu \nu}\right]$. It is readily seen that $i\left[p_{m}, M_{n-}\right]=\delta_{m n} p_{-} \neq 0$ (for $m=n$ ), therefore $M_{n-}$ does not belong to the little group of $p_{\mu}$ either. We are left with the generators $\left\{M_{m n}, M_{+n}\right\}$ which preserve the (vanishing) value of $p_{+}$. It turns out to be more convenient for later purpose to work with the generators $\pi_{n}:=p_{-} M_{+n}=p^{\mu} M_{\mu n}$ instead. This redefinition does not modify the algebra since $p_{-}$commutes with all the generators of the little group. From the Poincaré algebra (1)-(3) one finds, in the light-cone frame,

$$
\begin{aligned}
i\left[M_{m n}, M_{p q}\right] & =\delta_{n p} M_{m q}-\delta_{m p} M_{n q}-\delta_{q m} M_{p n}+\delta_{q n} M_{p m}, \\
i\left[\pi_{m}, M_{n p}\right] & =\delta_{m n} \pi_{p}-\delta_{m p} \pi_{n}, \\
i\left[\pi_{m}, \pi_{n}\right] & =0 .
\end{aligned}
$$

As can be seen, the generators $\left\{M_{m n}, \pi_{m}\right\}$ span the Lie algebra of the inhomogeneous orthogonal group $I S O(D-2)$.

For later purpose, notice that the quadratic Casimir operator of the Euclidean algebra $\mathfrak{i s o}(D-2)$ presented by the generators $\left\{M_{m n}, \pi_{m}\right\}$ and the relations (14)-(16) is the square of the "translation" generators

$$
\mathcal{C}_{2}(\mathfrak{i s o}(D-2))=\pi^{m} \pi_{m}
$$


To end up this discussion, one should consider the case of a vanishing momentum. Of course, the orbit of a vanishing vector under linear transformations is itself while its stabilizer is the whole linear subgroup. Therefore, the subgroup of $S O(D-1,1)^{\uparrow}$ leaving invariant the zero-momentum vector $p^{\mu}=0$ is the whole group itself. This ends up the determination of the orbit and stabilizer of any possible vector $\in \mathbb{R}^{D-1,1}$.

Remark: The zero-momentum $\left(q^{\mu}=0\right)$ representations are essentially UIRs of the little group $S O(D-1,1)^{\uparrow}$ because the translation group acts trivially. The proper orthochronous Lorentz group may be identified with the isometry group of the de Sitter spacetime $d S_{D-1}$. In other words, the wave function of the zero-momentum representation may be interpreted as a wave function on a lower-dimensional de Sitter spacetime, and conversely. Even though their physical meaning may differ, both UIRs may be mathematically identified.

\subsection{Classification}

To summarize the previous subsection, the UIRs of the Poincaré group $I S O(D-1,1)^{\uparrow}$ have been divided into four classes according to the four possible orbits of the momentum, summarized in the following table (where $m^{2}>0$ ):

\begin{tabular}{|c|c|c|c|}
\hline Gender & Orbit & Stability subgroup & UIR \\
\hline \hline$p^{2}=-m^{2}$ & Mass-shell & $S O(D-1)$ & Massive \\
\hline$p^{2}=0$ & Light-cone & $I S O(D-2)$ & Massless \\
\hline$p^{2}=+m^{2}$ & Hyperboloid & $S O(D-2,1)^{\uparrow}$ & Tachyonic \\
\hline$p_{\mu}=0$ & Origin & $S O(D-1,1)^{\uparrow}$ & Zero-momentum \\
\hline
\end{tabular}

The problem of classifying the UIRs of the Poincaré group $\operatorname{ISO}(D-1,1)^{\uparrow}$ has been reduced to the classication of the UIRs of the stability subgroup of the momentum, which are either a unimodular orthogonal group, an Euclidean group or a proper orthochronous Lorentz group.

Actually, the method of induced representation may also be applied to the classification of the UIRs of the Euclidean group ISO $(D-2)$, the little group of a massless particle. The important thing to understand is that the light-like momentum $p^{\mu}$ is fixed and that what should be examined is the action of the little group on the physical components characterized by $\sigma$. From (16) one sees that the $D-2$ "translation" generators $\pi^{i}$ commute with each other, so it is natural to express physical states $\Psi_{p, \sigma}$ in terms of eigenvectors $\xi^{m}$ of these generators $\pi^{m}$. Introducing a label $\varsigma$ to denote all remaining physical components, one thus considers states $\Psi_{p, \xi, \varsigma}$ with $\pi_{m} \Psi_{p, \xi, \zeta}=\xi_{m} \Psi_{p, \xi, \zeta}$. The discussion presented in Subsection 3.1 may be repeated almost identically, up to the replacement of the momentum $p$ by the eigenvector $\xi$, the label $\sigma$ by $\varsigma$, the Poincaré group $\operatorname{ISO}(D-1,1)^{\uparrow}$ by the Euclidean group $\operatorname{ISO}(D-2)$ and the proper orthochronous Lorentz group $S O(D-1,1)^{\uparrow}$ by the unimodular orthogonal group $S O(D-2)$. The conclusion is therefore similar: the problem of determining all possible irreps of the massless little group $I S O(D-2)$ has been reduced to the problem of finding all possible irreps of the stability subgroup of the $(D-2)$-vector $\xi$, called the short little group in the literature [8].

The massless representations induced by a non-trivial representation of the little group may therefore be divided into distinct categories, depending on the class of momentum to which $\xi^{m}$ belongs. The situation is simpler here because there exist only two possible classes of orbits for a vector in the Euclidean space $\mathbb{R}^{D-2}$ : either the origin $\xi^{m}=0$, or a $(D-3)$ sphere of radius $\mu>0$. In the first case, the action of the elusive "translation" operators $\pi^{m}$ is trivial and, effectively, the little group is identified with the short little group $S O(D-2)$. These representations are most often referred to as helicity representations by analogy with the $D=4$ case. In the second case, the corresponding representations are most often referred to as 
continuous spin representations [8], even though Wigner also used the name infinite spin [7]. The former name originates from the fact that the transverse vector $\xi^{m}$ has a continuous range of values. Nevertheless, the latter name is more adequate in some respect, as will be argued later on. Roughly speaking the point is that, on the orbit $\xi^{2}=\mu^{2}$, the components spanned by the internal vector $\xi^{m}$ take values on the sphere $S^{D-3} \subset \mathbb{R}^{D-2}$ of radius $\mu=|\xi|$. The "radius" $\mu$ of this internal sphere has actually the dimension of a mass parameter (the reason is that the sphere $S^{D-3}$ is somehow in internal "momentum" space). Indeed, for massless representations, the parameter $\mu$ classifying the various irreps should be understood as the analogue of the mass for massive irreps, while the angular coordinates on the sphere $S^{D-3}$ are the genuine "spin" degrees of freedom, the Fourier conjugates of which are discrete variables as is more usual for spin degrees of freedom. This point of view motivates the name "infinite spin." ${ }^{3}$

To summarize, the UIRs of the Euclidean group $I S O(D-2)$ are divided into two classes according to the two possible orbits of the $(D-2)$-vector $\xi_{m}$, summarized in the following table:

\begin{tabular}{|c|c|c|c|}
\hline Gender & Orbit & Stability subgroup & Massless UIR \\
\hline \hline$\xi^{2}=\mu^{2}$ & Sphere & $S O(D-3)$ & Infinite spin \\
\hline$\xi_{m}=0$ & Origin & $S O(D-2)$ & Helicity \\
\hline
\end{tabular}

As a consequence of the method of induced representations, the physical components of a first-quantized elementary particle span a UIR of the little group. The number of local degrees of freedom (or of physical components) of the field theory is thus given by the dimension of the Hilbert space carrying the UIR of the little group. In the light of the standard results of representation theory (reviewed in Subsection 1.3) and using the method of induced representation, the UIRs of the Poincaré group may alternatively be divided into two distinct classes: the finite-component ones (the massive and the helicity reps) for which the (short) little group is compact, and the infinite-component ones (the infinite-spin, the tachyonic and the zero-momentum reps) for which the little group is non-compact.

\section{Remarks:}

- More precisely, the lower-dimensional cases $D=2,3$ are degenerate in the following sense (when $p^{\mu} \neq 0$ ). In $D=2$, all little groups are trivial, thus all physical fields are scalars. In $D=3$, all little groups are Abelian (massive: $S O(2)$, massless: $\mathbb{R}$, tachyonic: $S O(1,1)^{\uparrow} \cong \mathbb{R}$ ) hence all their UIRs have (complex) dimension one: generically, fields have one physical degrees of freedom. Notice that the helicity reps may be assigned a "conformal spin" if they are extended to irreps of the group $S O(D, 2) \supset S O(D-1,1)^{\uparrow}$. Notice also that the "spin" of a massive representation is not discretized in $D=3$ but can be an arbitrary real number ${ }^{4}$ [10] because the universal cover of $S O(2,1)^{\uparrow}$ covers it infinitely often.

- For massive and helicity representations, the number of local physical degrees of freedom may be determined from the well known formulas for the dimension of any UIR of the orthogonal groups (reviewed in Subsection 4.3 for the tensorial irreps).

- This group-theoretical analysis does not probe topological theories (such as Chern-Simons theory) because such theories correspond to identically vanishing representations of the little group since they have no local physical degrees of freedom.

The following corollary provides a group-theoretical explanation of the fact that combining the principle of relativity with the rules of quantum mechanics necessarily leads to field theory.

\footnotetext{
${ }^{3}$ Actually, in Subsection 5.3 an explicit derivation of the continuous spin representation from a proper "infinite spin" limit of a massive representation is reviewed. All the former comments find their natural interpretation in this point of view.

${ }^{4}$ This peculiarity is related to the existence of anyons in three spacetime dimensions, cf. Appendix B.
} 
Corollary: Every non-trivial unitary irreducible representation of the isometry group of any maximally-symmetric spacetime is infinite-dimensional.

Proof: The Hilbert space carrying a non-trivial unitary representation of the Poincaré group is infinite-dimensional because (i) in the generic case, $q_{\mu} \neq 0$, the orbit is either a hyperboloid $\left(p^{2} \neq 0\right)$ or a cone $\left(p^{2}=0\right)$ and the space of wave functions on the orbit is infinite-dimensional, (ii) the zero-momentum representations of the Poincaré group are unitary representations of the de Sitter isometry group. Thus, the proof is ended by noticing that all non-trivial unitary representations of the isometry group of (anti) de Sitter spacetimes $(A) d S_{D}$ also are infinitedimensional, because their isometry groups are pseudo-orthogonal Lie groups.

\section{Tensorial representations and Young diagrams}

Most of the material reviewed here may be found in textbooks such as [11-13]. Nevertheless, large parts of this section are either copied or adapted from the reference [14] because altogether it provides an excellent summary, both for its pedagogical and comprehensive values. The material collected in the present section goes slightly beyond what is strictly necessary for these lectures, but the reader may find it useful in specific applications.

\subsection{Symmetric group}

An (unlabeled) Young diagram, consisting of $n$ boxes arranged in $r$ (left justified) rows, represents a partition of the integer $n$ into $r$ parts:

$$
n=\sum_{a=1}^{r} \lambda_{a}, \quad\left(\lambda_{1} \geqslant \lambda_{2} \geqslant \ldots \geqslant \lambda_{r}\right) .
$$

That is, $\lambda_{a}$ is the number of boxes in the $a$ th row. Successive row lengths are non-increasing from top to bottom. A simpler notation for the partition is the list of its parts: $\lambda=\left\{\lambda_{1}, \lambda_{2}, \ldots\right.$, $\left.\lambda_{r}\right\}$. For instance,

$$
\{3,3,1\}=\begin{array}{|l|l}
\square & \square \\
\hline \square & \square
\end{array}
$$

Examples: There are five partitions of 4:

$$
\{4\},\{3,1\},\{2,2\},\{2,1,1\},\{1,1,1,1\} \text {. }
$$

Partitions play a key role in the study of the symmetric group $\mathfrak{S}_{n}$. This is the group of all permutations of $n$ objects. It has $n$ ! elements and its inequivalent irreducible representations may be labeled by the partitions of $n$. [In the following, Greek letters $\lambda, \mu$ and $v$ will be used to specify not only partitions and Young diagrams but also irreducible representations of the symmetric group and other groups.]

The connection between the symmetric group and tensors was initially developed by $\mathrm{H}$. Weyl. This connection can be approached in (at least) two equivalent ways.

A. Let $T_{\mu_{1} \ldots \mu_{n}}$ be a 'generic' $n$-index tensor, without any special symmetry property. [For the moment, 'tensor' just means a function of $n$ indices, not necessarily with any geometrical realization. It must be meaningful, however, to add - and form linear combinations of - tensors of the same rank.] A Young tableau, or labeled Young diagram, is an assignment of the numbers $1,2, \ldots, n$ to the $n$ boxes of a Young diagram $\lambda$. The tableau 
is standard if the numbers are increasing both along rows from left to right and down columns from top to bottom. The entries $1, \ldots, n$ in the tableau indicate the $n$ successive indices of $T_{\mu_{1} \ldots \mu_{n}}$. The tableau defines a certain symmetrization operation on these indices: symmetrize on the set of indices indicated by the entries in each row, then antisymmetrize the result on the set of indices indicated by the entries in each column. The resulting object is a tensor, $\widetilde{T}$, with certain index symmetries. Now let each permutation of $\mathfrak{S}_{n}$ act (separately) upon $\widetilde{T}$. The $n$ ! results are not linearly independent; they span a vector space $V_{\lambda}^{\mathfrak{S}_{n}}$ which supports an irreducible representation of $\mathfrak{S}_{n}$. Different tableaux corresponding to the same diagram $\lambda$ yield equivalent (by not identical) representations.

Example: The partition $\{2,2\}$ of 4 has two standard tableaux:

\begin{tabular}{|l|l|l|l|l|}
\hline 1 & 2 \\
\hline 3 & 4
\end{tabular}$\quad$ and $\quad$\begin{tabular}{rl|l}
1 & 3 \\
\hline 2 & 4 \\
\hline
\end{tabular}

Let us construct the symmetrized tensor $\widetilde{T}_{a b c d}:=R_{a b \mid c d}$ corresponding to the second of these:

$$
\begin{array}{|l|l|}
\hline \mathrm{a} & \mathrm{c} \\
\hline \mathrm{b} & \mathrm{d} \\
\hline
\end{array}
$$

First symmetrize over the first and third indices ( $a$ and $c$ ), and over the second and fourth $(b$ and $d)$ :

$$
\frac{1}{4}\left(T_{a b c d}+T_{c b a d}+T_{a d c b}+T_{c d a b}\right) .
$$

Now antisymmetrize the result over the first and second indices ( $a$ and $b$ ), and over the third and fourth ( $c$ and $d) ;{ }^{5}$ dropping the combinatorial factor $\frac{1}{16}$, we get

$$
\begin{aligned}
R_{a b \mid c d} & =T_{a b c d}+T_{c b a d}+T_{a d c b}+T_{c d a b}-T_{b a c d}-T_{c a b d}-T_{b d c a}-T_{c d b a} \\
& -T_{a b d c}-T_{d b a c}-T_{a c d b}-T_{d c a b}+T_{b a d c}+T_{d a b c}+T_{b c d a}+T_{d c b a} .
\end{aligned}
$$

It is easy to check that $R$ possesses the symmetries of the Riemann tensor. There are two independent orders of its indices (e.g. $R_{a b \mid c d}$ and $R_{a c \mid b d}$ ), and applying any permutation to the indices produces some linear combination of those two basic objects. On the other hand, performing on $T$ the operations prescribed by the first tableau in (19) produces a different expression $P_{a c \mid b d}$, which, however, generates a two-dimensional representation of $\mathfrak{S}_{4}$ with the same abstract index structure as that generated by $R$. A non-standard tableau would also yield such a representation, but the tensors within it would be linear combinations of those already found. One finds

$$
\begin{aligned}
P_{a c \mid b d} & =T_{a b c d}+T_{b a c d}+T_{a b d c}+T_{b a d c}-T_{c b a d}-T_{b c a d}-T_{c b d a}-T_{b c d a} \\
& -T_{a d c b}-T_{d a c b}-T_{a d b c}-T_{d a b c}+T_{c d a b}+T_{d c a b}+T_{c d b a}+T_{d c b a} .
\end{aligned}
$$

As the reader may check, no linear combinations of $P$ can reproduce $R$. The objects $P_{a b \mid c d}, P_{a c \mid b d}, R_{a b \mid c d}$ and $R_{a c \mid b d}$ are linearly independent. Although $R$ and $P$ are characterized by the same Young diagram, they are associated with different standard Young tableaux and therefore span two different (although equivalent) irreducible representations of $\mathfrak{S}_{n}$. Two representations may indeed be equivalent without being identical. This happens in particular for the irreducible decomposition of the regular representation of $\mathfrak{S}_{n}$ where every irreducible representation appears with a multiplicity equal to

\footnotetext{
${ }^{5}$ Here we adopt the convention that the second round of permutations interchanges indices with the same names, rather than indices in the same positions in the various terms. The opposite convention is tantamount to antisymmetrizing first, which leads to a different, but mathematically isomorphic, development of the representation theory. The issue here is analogous to the distinction between space-fixed and body-fixed axes in the study of the rotation group (active or passive transformations).
} 
its dimension. When the dimension of an $\mathfrak{S}_{n}$ irreducible representation is $d>1$, then $d$ copies of that irreducible representation appear in the decomposition of the regular representation of $\mathfrak{S}_{n}$ and all these $d$ representations are equivalent, although not identical.

Example: Define a symmetrized Riemann tensor (the Jacobi tensor) by $J_{a d ; b c}:=\frac{1}{2}\left(R_{a b \mid c d}\right.$ $\left.+R_{a c \mid b d}\right)$. It obeys $J_{a b ; c d}=J_{b a ; c d}=J_{a b ; d c}$. Then it is easy to show that $R_{a b \mid c d}=\frac{2}{3}\left(J_{a d ; b c}\right.$ $\left.-J_{b d ; a c}\right)$. Thus the tensor $J$ has no fewer independent components and contains no less information than the tensor $R$, despite the extra symmetrization; $R$ is recovered from $J$ by an antisymmetrization. The tensors $R$ and $J$ are really the same tensor expressed with respect to different bases.

B. The regular representation of $\mathfrak{S}_{n}$ is the $n$ !-dimensional representation obtained by letting $\mathfrak{S}_{n}$ act by left multiplication on the formal linear combinations of elements of $\mathfrak{S}_{n}$. [That is, one labels the basis vectors of $\mathbb{R}^{n !}$ by elements of $\mathfrak{S}_{n}$, defines that action of each permutation on the basis vectors in the natural way, and extends this definition to the whole space by linearity.] Equivalently, the vector space of the regular representation is the space of real-valued functions defined on $\mathfrak{S}_{n}$. [In general the regular representation is defined with complex scalars, but for $\mathfrak{S}_{n}$ it is sufficient to work with real coefficients.]

Regular representation: The regular representation contains every irreducible representation with a multiplicity equal to its dimension. Each Young diagram $\lambda$ corresponds to an irreducible representation of $\mathfrak{S}_{n}$. Its dimension and multiplicity are equal to the number of standard tableaux of diagram $\lambda$.

The symmetrization procedure described under A. can be transcribed to the more abstract context B. to construct a projection operator onto the subspace of $\mathbb{R}^{n !}$ supporting each representation. [The numerical coefficient needed to normalize the tableau operation as a projection - an operator whose square is itself - is not usually the same as that accumulated from the individual symmetrization operations. For example, to make $R_{a b c d}$ into a projection of $T_{a b c d}$, one needs to divide by 12 , not 16.$]$

Example: In (18), the partition $\{4\}$ corresponds to the totally symmetric four-index tensors, a one-dimensional space $V_{\{4\}}^{\mathfrak{S}_{4}}$. Similarly, $\{1,1,1,1\}$ yields the totally antisymmetric tensors. A generic rank-four tensor, $T_{a b c d}$, can be decomposed into the sum of its symmetric and antisymmetric parts, plus a remainder. The theory we are expounding here tells how to decompose the remainder further. The partition $\{2,2\}$ yields two independent two-dimensional subrepresentations of the regular representation; in more concrete terms, there are two independent pieces of $T_{a b c d}\left(\frac{1}{12} R_{a b \mid c d}\right.$ and $\left.\frac{1}{12} P_{a c \mid b d}\right)$ constructed as described in connection with (19). One of these $\left(R_{a b \mid c d}\right)$ has exactly the symmetries of the Riemann tensor; the other $\left(P_{a c \mid b d}\right.$, coming from the first tableau of (19)) has the same abstract symmetry as the Riemann tensor, but with the indices ordered differently. Finally, each of the remaining partitions in (18), i.e., $\{3,1\}$ and $\{2,1,1\}$, can be made into a standard tableau in three different ways. Therefore, each of these two representations has three separate pieces of $T$ corresponding to it, and each piece is threedimensional (has three independent index orders after its symmetries are taken into account). Thus the total number of independent tensors which can be formed from the irreducible parts of $T_{a b c d}$ by index permutations is

$$
1^{2}+1^{2}+2^{2}+3^{2}+3^{2}=24=4 !
$$

which is simply the total number of permutations of the indices of $T$ itself, as it must be.

To state a formula for the dimension of an irreducible representation $V_{\lambda}^{\mathfrak{S}_{n}}$ of $\mathfrak{S}_{n}$, we need the concept of the hook length of a given box in a Young diagram $\lambda$. The hook length of a box 
in a Young diagram is the number of squares directly below or directly to the right of the box, including the box once:

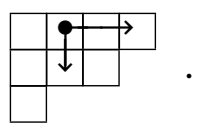

Example: In the following diagram, each box is labeled by its hook length:

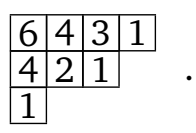

One then has the following hook length formula for the dimension of the representation $V_{\lambda}^{\mathfrak{S}_{n}}$ of $\mathfrak{S}_{n}$ corresponding to the Young diagram $\lambda$ :

$$
\operatorname{dim} V_{\lambda}^{\mathfrak{S}_{n}}=\frac{n !}{\prod(\text { hook lengths })} .
$$

Remark: Note carefully that the "dimension" we have been discussing up to now is the number of independent index orders of a tensor, not the number of independent components when the tensor is realized geometrically with respect to a particular underlying vector space or manifold. The latter number depends on the dimension (say $D$ ) of that underlying space, while the former is independent of $D$ (so long as $D$ is sufficiently large, as we tacitly assume in generic discussions). For example, the number of components of an antisymmetric two-index tensor is $\frac{D(D-1)}{2}$, but the number of its index orders is always 1 , except in dimension $D=1$ where no non-zero antisymmetric tensors exist at all.

\subsection{General linear group}

We now turn to the representation theory of the general linear and orthogonal groups, where the (spacetime) dimension $D$ plays a key role. The theory of partitions and of the representations of the permutation groups is the foundation on which this topic is built.

Let $\left\{v_{a}\right\}$ represent a generic element of $\mathbb{R}^{D *}$ (or of the cotangent space at a point of a $D$-dimensional manifold). The action of non-singular linear operators on this space gives a $D$ dimensional irreducible representation $V \cong \mathbb{R}^{D *}$ of the general linear group $G L(D)$; indeed, this representation defines the group itself. The rank-two tensors, $\left\{T_{a b}\right\}$, carry a larger representation of $G L(D)\left(V \otimes V\right.$, of dimension $\left.D^{2}\right)$, where the group elements act on the two indices simultaneously. The latter representation is reducible: it decomposes into the subspace of symmetric and antisymmetric rank-two tensors $V \otimes V \cong(V \odot V) \oplus(V \wedge V)$, of respective dimensions $\frac{D(D+1)}{2}$ and $\frac{D(D-1)}{2}$. Similarly, the tensor representation of rank $n, V^{\otimes n}$, decomposes into irreducible representations of $G L(D)$ which are associated with the irreducible representations of $\mathfrak{S}_{n}$ acting on the indices, which in turn are labeled by the partitions of $n$, hence by Young diagrams. Young diagrams with more than $D$ rows do not contribute [if $\lambda$ is a partition of $n$ into more than $D$ parts, then the associated index symmetrization of a $D$-dimensional rank- $n$ tensor yields an expression that vanishes identically; in particular, there are no non-zero totally antisymmetric rank- $n$ tensors if $n>D]$.

More precisely, let $\lambda$ be a Young tableau. The Schur module $V_{\lambda}^{G L(D)}$ is the vector space of all rank- $n$ tensors $\tilde{T}$ in $V^{\otimes n}$ such that:

(i) the tensor $\tilde{T}$ is completely antisymmetric in the entries of each column of $\lambda$,

(ii) complete antisymmetrization of $\tilde{T}$ in the entries of a column of $\lambda$ and another entry of $\lambda$ that is on the right-hand side of the column vanishes. 
This construction is equivalent to the construction $\mathbf{A}$.

Example: Associated with the Young tableau (20), the tensor $R_{a b \mid c d}$ introduced in the subsection 4.1 obeys to the conditions (i) and (ii): $R_{a b \mid c d}=-R_{b a \mid c d}=-R_{a b \mid d c}$ and $R_{a b \mid c d}+R_{b c \mid a d}+$ $R_{c a \mid b d}=0$.

As explained in the footnote 5 , if one interchanges everywhere in the previous constructions the words "symmetric" and "antisymmetric," then the (reducible) representation spaces characterized by the same Young diagram [but not by the same Young tableau] are isomorphic and the conditions (i)-(ii) must be replaced with:

(a) the tensor is completely (or totally) symmetric in the entries of each column of $\lambda$,

(b) complete symmetrization of the tensor in the entries of a row of $\lambda$ and another entry of $\lambda$ that sits in a lower row vanishes.

Example: Taking the standard Young tableau (20) and constructing, following the "manifestly symmetric convention", the irreducible tensor associated with it, one obtains a tensor $\mathcal{R}$ with the same abstract index symmetries as $J$ [i.e. obeying the constraints $(a)$ and $(b)$ ] but which is however linearly independent from $J$, thence linearly independent from $R$ alone. The tensor $\mathcal{R}$ can be expressed as a linear combination of both $R$ and $P$. Similarly, taking the first standard Young tableau in (19) and following the manifestly symmetric convention, one obtains a tensor $\mathcal{P}$ obeying $(a)$ and $(b)$. This tensor is linearly independent from $P$ alone as it is a linear combination of both $P$ and $R$. Summarizing, associated with the Young diagram $\{2,2\}$ we have the (reducible) representation space spanned by either $\{R, P\}$ in the manifestly antisymmetric convention or by $\{\mathcal{R}, \mathcal{P}\}$ in the manifestly symmetric convention.

\section{Remarks:}

- An important point to note is that, by the previous construction featuring irreducible tensors with definite symmetry properties, one generates essentially all the finite-dimensional irreducible representations of $G L(D, \mathbb{R})$. To be more precise, $G L(D, \mathbb{R})$ tensors can be of type $(p, q)$, i.e., having $p$ contravariant indices and $q$ covariant ones. The exhaustive list of finitedimensional irreducible representations of $G L(D, \mathbb{R})$ is provided by $(p, q)$-type tensors characterised by a pair of Young tableaux of rank $p$ and $q$, respectively, and such that the contraction of any covariant index with a contravariant one gives zero identically. See e.g. Chapter 13 of [9] for more details.

- In order to make contact with an alternative road to the representation theory of $G L(D)$, one says that the irreducible representation $\Gamma_{\lambda^{1}} \ldots \lambda^{D-1}$ of $\mathfrak{s l}(D, \mathbb{C}) \equiv A_{D-1}$ with highest weight $\Lambda=\lambda^{1} \Lambda_{(1)}+\lambda^{2} \Lambda_{(2)}+\ldots+\lambda^{D-1} \Lambda_{(D-1)}$ [see e.g. the Part II of the lecture notes [3] for definitions and notations] is obtained by applying the Schur functor $\mathbb{S}_{\lambda}$ [i.e. the construction presented above] to the standard representation $V$, where the Young diagram is

$$
\lambda=\left\{\lambda^{1}+\ldots+\lambda^{D-1}, \lambda^{2}+\ldots+\lambda^{D-1}, \ldots, \lambda^{D-1}, 0\right\} .
$$

In terms of the Young diagram for $\lambda$, the Dynkin labels $\lambda^{a}(1 \leqslant a \leqslant D-1)$ are the differences of lengths of rows: $\lambda^{a}=\lambda_{a}-\lambda_{a+1}$.

Example: If $D=6$, then

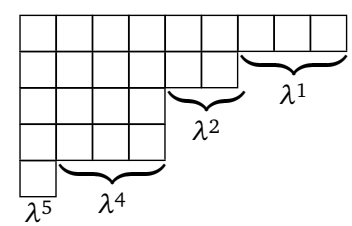


is the Young diagram corresponding to the irrep $\Gamma_{3,2,0,3,1}$ of $A_{5} \equiv \mathfrak{s l}(6, \mathbb{C})$. $\lambda$ is:

The dimension of the representation $V_{\lambda}^{G L(D)}$ of $G L(D)$ corresponding to the Young diagram

$$
\operatorname{dim} V_{\lambda}^{G L(D)}=\prod \frac{D-\text { row }+ \text { column }}{\text { hook length }}
$$

where the product is over the $n$ boxes while "row" and "column" respectively give the place of the corresponding box. As was underlined before, the formula (22) is distinct from the hook length formula (21).

Examples:

- In the following diagram

$$
\begin{aligned}
& \begin{array}{|l|l|l|l|}
\hline 5 & 6 & 7 & 8 \\
\hline
\end{array} \\
& \begin{array}{|l|l|l|l|}
\hline 5 & 6 & 7 & 8 \\
\hline 4 & 5 & 6 \\
\hline
\end{array} \\
& 3
\end{aligned}
$$

each box is labeled by its value in the numerator of (22) for $D=5$. Observe that, for the corresponding diagram $\lambda, \operatorname{dim} V_{\lambda}^{G L(5)}=1050 \neq 70=\operatorname{dim} V_{\lambda}^{\mathfrak{S}_{8}}$.

- The space of (anti)symmetric tensors of $V$ of rank $n$ are denoted by $\odot^{n}(V)$ (respectively, $\wedge^{n}(V)$ ). It carries an irreducible representation of $G L(D)$ labeled by a Young diagram made of one row (respectively, column) of length $n$. The dimensions

$$
\operatorname{dim} \odot^{n}(V)=\left(\begin{array}{c}
D+n-1 \\
n
\end{array}\right), \quad \operatorname{dim} \wedge^{n}(V)=\left(\begin{array}{l}
D \\
n
\end{array}\right),
$$

are easily computed from the formula (22) and reproduce the standard results obtained from combinatorial arguments.

If $T_{1}$ and $T_{2}$ are tensors of ranks $n_{1}$ and $n_{2}$, respectively, then their tensor product is a tensor of rank $n_{1}+n_{2}$. Each factor $T_{j}$ transforms under index permutation according to some representation of $\mathfrak{S}_{n_{j}}$, and under linear transformation by the corresponding representation of $G L(D)$. It follows immediately that the tensor product $T_{1} \otimes T_{2}$ transforms as some representation of $\mathfrak{S}_{n_{1}} \times \mathfrak{S}_{n_{2}}$. This induces a representation of the full permutation group $\mathfrak{S}_{n_{1}+n_{2}}$ which is associated with a corresponding representation of $G L(D)$. It is possible to reduce these last two representations into a sum of irreducible ones. We may assume that the factor representations are irreducible, since the original tensors $T_{j}$ could have been broken into irreducible parts at the outset.

Littlewood-Richardson rule: The decomposition of an "outer product" $\mu \cdot v$ of irreducible representations $\mu$ and $v$ of $\mathfrak{S}_{n_{1}}$ and $\mathfrak{S}_{n_{2}}$, respectively, into irreducible representations of $\mathfrak{S}_{n_{1}+n_{2}}$ can be determined by means of the following algorithm involving Young diagrams. The product is commutative, so it does not matter which factor is regarded as the "right-hand" one. [In practice, on should choose the simpler Young diagram for that role.]

(I) Label each box in the top row of the right-hand diagram, $v$, by "a", each box in the second row by " $b$ ", etc.

(II) Add the labeled boxes of $v$ to the left-hand diagram $\mu$, one at a time, first the as, then the $b s, \ldots$, subject to these constraints:

(A) No two boxes in the same column are labeled with the same letter;

(B) At all stages the result is a legitimate Young diagram; 
(C) At each stage, if the letters are read right-to-left along the rows, from top to bottom, one never encounters more $b$ s than $a$ s, more $c s$ than $b$ s, etc.

(III) Each of the distinct diagrams constructed in this way specifies an irreducible subrepresentation $\lambda$, appearing in the decomposition of the outer product. The same labeled Young diagram may arise in more than one way; the multiplicity of that representation must be counted accordingly.

\section{Remarks:}

- This rule enables products of distinct tensors to be decomposed. When the factors are the same tensor, the list is further restricted by the requirement of symmetry under interchange of the factors. This is the problem of plethysm, whose solution requires more complicated techniques than the Littlewood-Richardson rule.

- Representations with too many parts (columns of length greater than $D$ ) must be deleted from the list of subrepresentations of the $G L(D)$. [If irreducible representations of the special linear group $S L(D)$ are considered instead, every column of length $D$ must be removed from the corresponding Young diagram.]

\subsection{Orthogonal group}

It remains to consider index contractions. Up to now we considered only covariant tensors, because in the intended application there is a metric tensor which serves to relate contravariant and covariant tensors. Contractions are mediated by this metric. Implicitly, therefore, one is restricting the symmetry group of the problem from the general linear group to the subgroup that leaves the metric tensor invariant, the orthogonal group $O(D)$. [If the metric has indefinite signature, the true symmetry group is a non-compact analogue of the orthogonal group, such as the Lorentz group. This does not affect the relevant aspects of the finite-dimensional representation theory.] Each irreducible $G L(D)$ representation $V_{\lambda}^{G L(D)}$ decomposes into irreducible $O(D)$ representations $V_{v}^{O(D)}$, labeled by Young diagrams $v$ obtained by removing an even number of boxes from $\lambda$. The branching rule for this process involves a sort of inverse of the Littlewood-Richardson rule:

Restriction from $G L(D)$ to $O(D)$ : The irreps of $G L(D)$ may be reduced to direct sums of irreps of $O(D)$ by extracting all possible trace terms formed by contraction with products of the metric tensor and its inverse.

The reduction is given by the branching rule for $G L(D) \downarrow O(D)$ :

$$
V_{\lambda}^{G L(D)}=V_{\lambda / \Delta}^{O(D)} \equiv V_{\lambda}^{O(D)} \oplus V_{\lambda /\{2\}}^{O(D)} \oplus V_{\lambda /\{4\}}^{O(D)} \oplus V_{\lambda /\{2,2\}}^{O(D)} \oplus \ldots,
$$

where $\Delta$ is the formal infinite sum [15]

$$
\Delta=1+\square+\square+\square \mid \square+\square+\ldots
$$

corresponding to the sum of all possible plethysms of the metric tensor, and where $\lambda / \mu$ means the sum of the Young diagrams $v$ such that $v \cdot \mu$ contains $\lambda$ according to the LittlewoodRichardson rule (with the corresponding multiplicity).

\section{Examples:}

- The $G L(D)$ irreducible representation labeled by the Young diagram $\{2,2\}$ decomposes with respect to $O(D)$ according to the direct sum $\{2,2\} / \Delta=\{2,2\}+\{2,0\}+\{0,0\}$ which corresponds to the decomposition of the Riemann tensor into the Weyl tensor, the traceless part of the Ricci tensor and the scalar curvature, respectively. 
- The $G L(D)$ irreducible representation labeled by the Young diagram $\{n\}$ decomposes with respect to $O(D)$ according to the direct sum $\{n\} / \Delta=\{n\}+\{n-2\}+\{n-4\}+\ldots$, corresponding to the decomposition of a completely symmetric tensor or rank $n$ into its traceless part, the traceless part of its trace, etc. This provides an alternative proof of the obvious fact that the number of independent components of a traceless symmetric tensor of rank $n$ is equal to the number of independent components of a symmetric tensor of rank $n$ minus the number of independent components of a symmetric tensor of rank $n-2$ (its trace): $\operatorname{dim} V_{\{n\}}^{O(D-2)}=\operatorname{dim} V_{\{n\}}^{G L(D)}-\operatorname{dim} V_{\{n-2\}}^{G L(D)}$. Using the formula (23) allows to show that

$$
\operatorname{dim} V_{\{n\}}^{O(D)}=\frac{(D+2 n-2)(D+n-3) !}{n !(D-2) !} .
$$

The very useful formula (25) contains as a particular case the well-known fact that all the traceless symmetric tensorial representations of $O(2)$ are two-dimensional (indeed, any UIR of an Abelian group is of complex dimension one). Moreover, the traceless symmetric tensorial representations of rank $n$ of the rotation group $O(3)$ are the well-known integer spin representations of dimension equal to $2 n+1$.

The following theorem is very important (see e.g. the first reference of [11-13]):

Vanishing irreps for (pseudo-)orthogonal groups: Whenever the sum of the lengths of the first two columns of a Young diagram $\lambda$ is greater than $D=p+q$, then the irreducible representation of $O(p, q)$ labeled by $\lambda$ is identically zero.

Young diagrams such that the sum of the lengths of the first two columns does not exceed $D$ are said to be allowed.

Finite-dimensional irreps of (pseudo-)orthogonal groups: Each non-zero finite-dimensional irreducible representation of $O(p, q)$ is isomorphic to a completely traceless tensorial representation, the symmetry properties of which are labeled by an allowed Young diagram $\lambda$.

The dimension of the tensorial irrep is determined by the following rule due to King [16]:

( $\alpha$ ) The numbers $D-1, D-3, D-5, \ldots, D-2 r+1$ are placed in the end boxes of the 1 st, 2 nd, 3rd, ..., $r$ th rows of the diagram $\lambda$. A labeled Young diagram of $n$ numbers is then constructed by inserting in the remaining boxes of the diagram, numbers which increase by one in passing from one box to its left-hand neighbor.

( $\beta$ ) This labeled Young diagram is extended to the limit of the triangular Young diagram $\tau$ of $r$ rows. This produces a Young diagram $\tilde{\lambda}$ the $a$ th row of which has length equal the maximum between the two integers $\tau_{a}=r-a+1$ and $\lambda_{a}$.

$(\gamma)$ The series of numbers in any row of the Young diagram $\tilde{\lambda}$ is then extended by inserting in the remaining boxes of the diagram, numbers which decrease by one in passing from one box to its right-hand neighbor. The resulting numbers will be called the "King length."

( $\delta$ ) The row lengths $\lambda_{1}, \lambda_{2}, \ldots, \lambda_{r}$ are then added to all of the numbers of the Young diagram $\tilde{\lambda}$ which lie on lines of unit slope passing through the first box of the 1 st, 2 nd, $\ldots, r$ th rows, respectively, of the Young diagram $\lambda$.

The dimension is equal to the product of the integers in the resulting labeled Young diagram $\widetilde{\lambda}$ divided by the product of

- the hook length of each box of $\lambda$, and of

- the King length of each box of $\tilde{\lambda}$ outside $\lambda$. 


\section{Examples:}

- In the following diagram, allowed for $D=5$,

$$
\begin{aligned}
& \begin{array}{|l|l|l|l|}
\hline 7 & 6 & 5 & 4 \\
\hline
\end{array} \\
& \begin{array}{|l|l|l|l|}
\hline & 6 & 5 & 4 \\
\hline 4 & 3 & 2 \\
\hline
\end{array} \\
& 0
\end{aligned}
$$

each box is labeled by its King length, while in the diagram

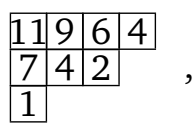

each box is labeled by the number obtained at the very end of King's rule. Observe that, for the corresponding diagram $\lambda$, it was not necessary to perform the steps $(\beta)-(\gamma)$ and that, $\operatorname{dim} V_{\lambda}^{O(5)}=231<1050=\operatorname{dim} V_{\lambda}^{G L(5)}$.

- In the following Young diagram $\lambda=\{2,2,1\}$, allowed for $D=5$,

$$
\begin{array}{|l|l|}
\hline 5 & 4 \\
\hline 3 & 2 \\
\hline 0 & \\
\hline
\end{array}
$$

each box is labeled by the number obtained after step $(\alpha)$. The step $(\beta)$ is now necessary and gives the Young diagram $\widetilde{\lambda}=\{3,2,1\}$. At the end of steps $(\gamma)$ and $(\delta)$, respectively, the result is

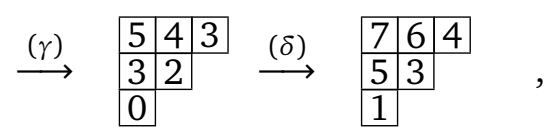

so that $\operatorname{dim} V_{\lambda}^{O(5)}=\frac{7 \cdot 6 \cdot 5 \cdot 4 \cdot 3}{(4 \cdot 3 \cdot 2) \cdot(3)}=35<75=\operatorname{dim} V_{\lambda}^{G L(5)}$.

- The space of traceless symmetric tensors of $V$ of rank $n$ carries an irreducible representation of $O(D)$ labeled by a Young diagram made of one row of length $n$ for which the dimension (25) is easily reproduced from the King rule, since the rules $(\beta)-(\gamma)$ may be omitted - Computing the number of components of the Weyl tensor and of a symmetric, traceless, ranktwo tensor in $D=4$ dimensions, enables one to give the decomposition $\{2,2\} / \Delta=\{2,2\}+$ $\{2,0\}+\{0,0\}$ of the Riemann tensor into the Weyl tensor, the traceless part of the Ricci tensor and the scalar curvature, respectively, in terms of the corresponding dimensions. This gives the well-known result $20=10+9+1$.

Unitary irreps of orthogonal groups: Each non-zero inequivalent UIR of $O(D)$ corresponds to an allowed Young diagram $\lambda$, and conversely.

Proof: The orthogonal group is compact, thence any UIR is finite-dimensional (see Subsection 1.3). Furthermore, any finite-dimensional irrep of the orthogonal group is labeled by an allowed Young diagram. Moreover, an important result is that any finite-dimensional representation may be endowed with a sesquilinear form which makes it unitary.

The quadratic Casimir operator of the orthogonal algebra $\mathfrak{s o}(D)$ presented by its generators and its commutation relations

$$
i\left[M_{\mu \nu}, M_{\rho \sigma}\right]=\delta_{\nu \rho} M_{\mu \sigma}-\delta_{\mu \rho} M_{v \sigma}-\delta_{\sigma \mu} M_{\rho v}+\delta_{\sigma v} M_{\rho \mu},
$$

is the sum of square of the generators (similarly to the definition (4) for $\mathfrak{s o}(D-1,1)$ since these two complex algebras are isomorphic). Its eigenvalue on a finite-dimensional irrep labeled by an allowed Young diagram $\lambda=\left\{\lambda_{1}, \lambda_{2}, \ldots, \lambda_{r}\right\}$ is given in the subsection 9.4.C of [2]:

$$
\left[\mathcal{C}_{2}(\mathfrak{s o}(D))-\sum_{a=1}^{r} \lambda_{a}\left(\lambda_{a}+D-2 a\right)\right] V_{\lambda}^{O(D)}=0
$$




\section{Examples:}

- The UIRs of the Abelian group $O(2) \cong U(1)$ are labeled by one integer only, which is the eigenvalue of the single generator on the irrep, say $h \in \mathbb{Z}$. The only allowed Young diagrams are made of a single row of length equal to the non-negative integer $s=|h|$. The traceless symmetric tensorial representations of $O(2)$ are two-dimensional, the sum of the two irreps labeled by $h= \pm s$. The formula (27) with $D=2, r=1$ and $\lambda_{1}=s$ gives the obvious eigenvalue $s^{2}$, since the quadratic Casimir operator of the rotation group $O(2)$ is equal to the square of the single generator.

- The quadratic Casimir operator of the rotation group $O(3)$ is the square of the angular momentum. The irrep of $O(3)$ with spin $s \in \mathbb{N}$ is labeled by the allowed Young diagram made of a single row of length equal to the integer $s$. The formula (27) with $D=3, r=1$ and $\lambda_{1}=s$ gives the celebrated eigenvalue $s(s+1)$.

- The irrep of $O(D)$ carried by the space of traceless symmetric tensors of rank $n$ is labeled by the allowed Young diagram $\{n\}$ made of a single row of length equal to an integer $n$. The formula (27) with $r=1$ and $\lambda_{1}=n$ gives the eigenvalue $n(n+D-2)$ for the quadratic Casimir operator.

The following branching rule is extremely useful in the process of dimensional reduction.

Restriction from $G L(D)$ to $G L(D-1)$ : The restriction to the subgroup $G L(D-1) \subset G L(D)$ of a finite-dimensional irrep of $G L(D)$ determined by the Young diagram $\lambda$ contains each irrep of $G L(D-1)$ labeled by Young diagrams $\mu$ such that

$$
\lambda_{1} \geqslant \mu_{1} \geqslant \lambda_{2} \geqslant \mu_{2} \geqslant \ldots \geqslant \mu_{r-1} \geqslant \lambda_{r} \geqslant \mu_{r} \geqslant 0,
$$

with multiplicity one. The same theorem holds for the restriction $O(D) \downarrow O(D-1)$ where $\lambda$ is an allowed Young diagram.

These rules are discussed in the section 8.8.A of [2]. They may be summarized in the following branching rule for $G L(D) \downarrow G L(D-1)$,

$$
V_{\lambda}^{G L(D)}=V_{\lambda / \Sigma}^{G L(D-1)} \equiv V_{\lambda}^{G L(D-1)} \oplus V_{\lambda /\{1\}}^{G L(D-1)} \oplus V_{\lambda /\{2\}}^{G L(D-1)} \oplus V_{\lambda /\{3\}}^{G L(D-1)} \oplus \ldots,
$$

where $\Sigma$ is the formal infinite sum of all Young diagrams made of a single row.

Example: The branching rule applied to symmetric irrep labeled by a Young diagram $\{n\}$ made of one row of length $n$ gives as a result:

$$
\{n\} / \Sigma=\{n\}+\{n-1\}+\{n-2\}+\ldots+\{1\}+\{0\} .
$$

This implies the obvious fact that a completely symmetric tensor of rank $n$ whose indices run over $D$ values may be decomposed as a sum of completely symmetric tensors of rank $n, n-1$, $\ldots, 1,0$ whose indices run over $D-1$ values. A non-trivial instance of the branching rule for $O(D) \downarrow O(D-1)$ is that the same result is true for traceless symmetric tensors as well.

\subsection{Auxiliary variables}

Let $\lambda$ be a Young diagram with $s$ columns and $r$ rows.

The Schur module $V_{\lambda}^{G L(D)}$ in the "manifestly antisymmetric convention" can be built via a convenient construction in terms of polynomials in $s \times D$ graded variables satisfying appropriate conditions. More precisely, the vector space $V_{\lambda}^{G L(D)}$ is isomorphic to a subspace of the associative algebra

$$
\mathcal{A}=\left(\otimes^{s} \wedge \mathbb{R}^{D *}\right) \otimes C^{\infty}\left(\mathbb{R}^{D}\right)=\otimes_{C^{\infty}\left(\mathbb{R}^{D}\right)}^{s} \Omega\left(\mathbb{R}^{D}\right)
$$


of $s$ tensor products of antisymmetric forms. The elements of $\mathcal{A}$ are called multiforms [17].

The $D$ generators of the $I$ th factor $\mathbb{R}^{D *}$ in $\left(\otimes^{s} \wedge \mathbb{R}^{D *}\right)$ are written $d_{I} x^{\mu}(\mu=0,1, \ldots, D-1)$. By definition, the multiform algebra $\mathcal{A}$ is presented by the graded commutation relations

$$
d_{I} x^{\mu} d_{J} x^{\nu}=(-)^{\delta} d_{I J} x^{v} d_{I} x^{\mu},
$$

where the wedge products are not written explicitly. The condition (i) of Subsection 4.2 is automatically verified for any element $\Phi \in \mathcal{A}$ due to the fact that the variables are anticommuting in a fixed column $(I=J)$. The $G L(D)$-irreducibility condition (ii) of Subsection 4.2 is implemented by the conditions

$$
\left(d_{I} x \cdot \frac{\partial^{L}}{\partial\left(d_{J} x\right)}-\delta_{I J} \ell_{I}\right) \Phi=0, \quad(I \leqslant J),
$$

where the dot stands for the contraction of the indices, $\ell_{I}$ for the length of the $I$ th column in the Young diagram $\lambda$ and $\partial^{L}$ stands for "left" derivative. By the Weyl construction, an element $\Phi \in \mathcal{A}$ satisfying (31) belongs to the Schur module $V_{\lambda}^{G L(D)}$. Following the discussion of Subsection 4.3, if $\lambda$ denotes an allowed Young diagram, such an element $\Phi \in V_{\lambda}^{G L(D)}$ is irreducible under the (pseudo)-orthogonal group $O(p, q)(p+q=D)$ if it is traceless, that is

$$
\left(\frac{\partial^{L}}{\partial\left(d_{I} x\right)} \cdot \frac{\partial^{L}}{\partial\left(d_{J} x\right)}\right) \Phi=0, \quad(\forall I, J),
$$

where the dot stands now for the contraction of indices via the use of the metric preserved by $O(p, q)$. An element $\Phi \in \mathcal{A}$ such that (31)-(32) are fulfilled belongs to the Schur module $V_{\lambda}^{O(p, q)}$ labeled by the Young diagram $\lambda$.

The Schur module $V_{\lambda}^{G L(D)}$ admits another convenient realization in terms of polynomials in $r \times D$ commuting variables. In other words, the vector space $V_{\lambda}^{G L(D)}$ is isomorphic to a subspace of the polynomial algebra in the variables $u_{a}^{\mu}(a=1,2, \ldots, r)$ where the index $a$ corresponds to each row. The condition (a) of Subsection 4.2 is automatically verified for any such polynomial due to the fact that the variables are commuting in a fixed row. The $G L(D)$-irreducibility condition (b) of Subsection 4.2 is implemented by the conditions

$$
\left(u_{a} \cdot \frac{\partial}{\partial u_{b}}-\delta_{a b} \lambda_{a}\right) \Phi=0, \quad(a \leqslant b),
$$

where the dot still stands for the contraction of the indices. The degree of homogeneity of the polynomial $\Phi$ in the variables $u_{a}^{\mu}$ (for fixed $a$ ) is $\lambda_{a}$. The corresponding coefficients are tensors irreducible under the general linear group. By the Weyl construction, a polynomial $\Phi\left(u_{a}\right)$ satisfying (33) belongs to the Schur module $V_{\lambda}^{G L(D)}$. Again, such an element $\Phi \in V_{\lambda}^{G L(D)}$ is irreducible under the (pseudo)-orthogonal group $O(p, q)(p+q=D)$ iff it is traceless, that is

$$
\left(\frac{\partial}{\partial u_{a}} \cdot \frac{\partial}{\partial u_{b}}\right) \Phi=0, \quad(\forall a, b),
$$

where the dot stands for the contraction of indices via the use of the metric preserved by $O(p, q)$. A polynomial $\Phi\left(u_{a}\right)$ such that (33)-(34) are fulfilled belongs to the Schur module $V_{\lambda}^{O(p, q)}$ labeled by an allowed Young diagram $\lambda$.

Example: Consider an irreducible representation of the orthogonal group $O(D)$ labeled by the Young diagram $\{n\}$ made of a single row of length equal to an integer $n$. The polynomial $\Phi(u) \in V_{\{n\}}^{O(D)}$ obeys to the irreducibility conditions

$$
\left(u \cdot \frac{\partial}{\partial u}-n\right) \Phi=0, \quad\left(\frac{\partial}{\partial u} \cdot \frac{\partial}{\partial u}\right) \Phi=0 .
$$


They mean that the polynomial is homogeneous (of degree equal to $n$ ) and harmonic, so that its components correspond to a symmetric traceless tensor of rank $n$ :

$$
\Phi(u)=\frac{1}{n !} \Phi_{\mu_{1} \ldots \mu_{n}} u^{\mu_{1}} \ldots u^{\mu_{n}}, \quad \delta^{\mu_{1} \mu_{2}} \Phi_{\mu_{1} \mu_{2} \mu_{3} \ldots \mu_{n}}=0 .
$$

Of course the integral of the square of such a polynomial over $\mathbb{R}^{D}$ is, in general, infinite. But the restriction of an harmonic polynomial on the unit sphere $\vec{u}^{2}=1$ is square integrable on $S^{D-1}$. This restriction is called a spherical harmonic of degree $n$. Therefore the space of spherical harmonics of degree $n$ provides an equivalent realization of the Schur module $V_{\{n\}}^{O(D)}$. For $D=3$, the space $V_{\{n\}}^{O(3)}$ is spanned by the usual spherical harmonics $Y_{n}^{m}(\theta, \phi)$ on the two-sphere with $|m| \leqslant n$.

\section{Remarks:}

- The infinitesimal generators of the pseudo-orthogonal group $O(p, q)$ are represented by the operators

$$
M_{\mu \nu}=i \sum_{a=1}^{r} u_{a}^{\rho}\left(g_{\rho \mu} \frac{\partial}{\partial u_{a}^{v}}-g_{\rho v} \frac{\partial}{\partial u_{a}^{\mu}}\right) .
$$

Reordering the factors and making use of (33)-(34) allows to reproduce the formula (27) for the eigenvalues of the quadratic Casimir operator.

- Instead of polynomial functions in the commuting variables, one may equivalently consider distributions obeying to the same conditions. The space of solutions would carry an equivalent irrep, as follows from the highest-weight construction of the representation. However, it does not make sense any more of talking about the "coefficients" of the homogeneous distribution so that the link with the equivalent tensorial representation is more intricate.

The example of the spherical harmonics suggests that it might be convenient to realize any unitary module of the orthogonal group $O(D)$ as a space of functions on the unit hypersphere $S^{D-1}$ satisfying some linear differential equations. Better, the symmetry under the orthogonal group would be made manifest by working with homogeneous harmonic functions on the ambient space $\mathbb{R}^{D}$, evaluated on any hypersphere $S^{D-1} \subset \mathbb{R}^{D}$.

Spherical harmonics: To any UIR of the isometry group $O(D)$ of a hypersphere $S^{D-1}$, one may associate manifestly covariant differential equations for functions on $S^{D-1}$ embedded in $\mathbb{R}^{D}$ whose space of solutions carry the corresponding UIR.

Proof: Any UIR of the isometry group $O(D)$ corresponds to a Schur module $V_{\lambda}^{O(D)}$ which may be realized as the space of polynomials $\Phi\left(\vec{u}_{a}\right)$ such that (33)-(34) are obeyed. Let us introduce the notation: $\vec{x}:=\vec{u}_{1}$ and $\vec{t}_{a-1}:=\vec{u}_{a}$ for $a=2, \ldots, r$. One interprets the polynomial $\Phi\left(\vec{x}, \vec{t}_{\underline{a}}\right)$ (where the index $\underline{a}$ runs from 1 to $r-1$ ) as a tensor field on the Euclidean space $\mathbb{R}^{D}$ parametrized by the Cartesian coordinates $\vec{x}$, with some auxiliary variables $\vec{t}_{\underline{a}}$ implementing the tensor components. The conditions (33)-(34) for $a$ and $b$ strictly greater than 1 imply that

$$
\left(t_{\underline{a}} \cdot \frac{\partial}{\partial t_{\underline{b}}}-\delta_{\underline{a b}} \lambda_{\underline{a}}\right) \Phi=0, \quad(\underline{a} \leqslant \underline{b}) \quad\left(\frac{\partial}{\partial t_{\underline{a}}} \cdot \frac{\partial}{\partial t_{\underline{b}}}\right) \Phi=0,
$$

where $\underline{\lambda}=\left\{\lambda_{2}, \ldots, \lambda_{r}\right\}$ is the Young diagram obtained from $\lambda$ by removing its first row. Thus the components of the "tensor field" $\Phi\left(\vec{x}, \vec{t}_{\underline{a}}\right)$ carry an irreducible representation of $O(D)$ labeled by $\underline{\lambda}$. The conditions (33) for $a=b=\overline{1}$ imply that

$$
\left(x \cdot \frac{\partial}{\partial x}-\lambda_{1}\right) \Phi=0,
$$


so the polynomial $\Phi\left(\vec{x}, \vec{t}_{a}\right)$ is homogeneous of degree $\lambda_{1}$ in the radial coordinate $|\vec{x}|$. The condition (34) for $a=b=\overline{1}$ is interpreted as the Laplace equation

$$
\left(\frac{\partial}{\partial x} \cdot \frac{\partial}{\partial x}\right) \Phi=0
$$

on the ambient space $\mathbb{R}^{D}$, it imples that the tensor field $\Phi$ is harmonic in ambient space. The condition (33) for $b>a=1$ states that the radial components vanish,

$$
\left(x \cdot \frac{\partial}{\partial t_{\underline{a}}}\right) \Phi=0,
$$

so the tensor components are longitudinal to the hyperspheres $S^{D-1}$. Therefore the evaluation of the non-vanishing components of $\Phi\left(\vec{x}, \vec{t}_{a}\right)$ on the unit hypersphere $|\vec{x}|=1$ is an intrinsic tensor field living on the hypersphere $S^{D-1}$ and whose tensor components carry an irrep of the stability subgroup $O(D-1)$ labeled by $\underline{\lambda}$. These tensor fields generalize the spherical harmonics to the generic case $r \geqslant 1$. Finally, the condition (34) for $b>a=1$ states that the tensor field is divergenceless in ambient space,

$$
\left(\frac{\partial}{\partial x} \cdot \frac{\partial}{\partial t_{\underline{a}}}\right) \Phi=0
$$

The differential equations (37) and (39) are written in ambient space but they may be reformulated in intrinsic terms on the hypersphere, at the price of losing the manifest covariance under the full isometry group $O(D)$.

\subsection{Euclidean group}

The method of induced representations was introduced in Subsection 3.1 for the Poincaré group $\operatorname{ISO}(D-1,1)^{\uparrow}$ and applied to the Euclidean group $\operatorname{ISO}(D-2)$ in Subsection 3.3. Focusing on the faithful (i.e. with a non-trivial action of the translation generators) irreps of the inhomogeneous orthogonal group, all of them are induced from an UIR of the stability subgroup. Using the results of the previous section 4.3, one may summarize the final result into the following classification.

Unitary irreps of the inhomogeneous orthogonal groups: Each inequivalent UIR of the group $I O(D)$ with a non-trivial action of its Abelian normal subgroup is associated with a positive real number $\mu$ and an allowed Young diagram of the subgroup $O(D-1)$, and conversely.

The orbits of the linear action of the orthogonal group $O(D)$ on the Euclidean space $\mathbb{R}^{D}$ are the hyperspheres $S^{D-1}$ of radius $R$. The isometry group of any such hypersphere $S^{D-1}$ is precisely $O(D)$. Considering a region of fixed size on these hyperspheres, in the limit $R \rightarrow \infty$ the sphere becomes a hyperplane $\mathbb{R}^{D-1}$. Therefore the homogeneous and inhomogeneous orthogonal groups are related by some infinite radius limit: $O(D) \rightarrow I O(D-1)$. Such a process is frequently referred to as an Inönü-Wigner contraction in the physics literature [18]. This is better seen at the level of the Lie algebra. Specializing the $D$ th directions, the commutation relations (26) take the form

$$
\begin{aligned}
i\left[M_{m n}, M_{p q}\right] & =\delta_{n p} M_{m q}-\delta_{m p} M_{n q}-\delta_{q m} M_{p n}+\delta_{q n} M_{p m}, \\
i\left[M_{m D}, M_{p q}\right] & =\delta_{m n} M_{p D}-\delta_{m p} M_{n D}, \\
i\left[M_{m D}, M_{p D}\right] & =M_{p m},
\end{aligned}
$$


where the latin letters take $D-1$ values. Defining $M_{m D}=R P_{m}$ and taking the limit $R \rightarrow \infty$ (with $P_{m}$ fixed) in the relations (40)-(42) lead to

$$
\begin{aligned}
i\left[M_{m n}, M_{p q}\right] & =\delta_{n p} M_{m q}-\delta_{m p} M_{n q}-\delta_{q m} M_{p n}+\delta_{q n} M_{p m} \\
i\left[P_{m}, M_{p q}\right] & =\delta_{m n} P_{p}-\delta_{m p} P_{n} \\
i\left[P_{m}, P_{p}\right] & =0 .
\end{aligned}
$$

As can be seen, the generators $\left\{M_{m n}, P_{m}\right\}$ span the Lie algebra of the inhomogeneous orthogonal group $I O(D-1)$. The former argument proves the contraction $\mathfrak{s o}(D) \rightarrow \mathfrak{i s o}(D-1)$.

The limit of a sequence of irreps of the homogeneous orthogonal group $O(D)$, in which one performs an Inönü-Wigner contraction, is automatically a representation of the inhomogeneous orthogonal group $I O(D-1)$ (if the limit is not singular). An interesting issue is the inverse problem: which irreps of $I O(D-1)$ may be obtained as the limit of such a sequence of irreps of $O(D)$ ? The problem is non-trivial because, generically, the limit of a sequence of irreps is a reducible representation.

Contraction of UIRs of the homogeneous orthogonal groups: Each inequivalent UIR of the group $I O(D-1)$ with a non-trivial action of its Abelian normal subgroup may be obtained as the contraction of a sequence of UIRs of the group $O(D)$.

More precisely, the Inönü-Wigner contraction $R \rightarrow \infty$ of a sequence of UIRs of $O(D)$, labeled by allowed Young diagrams $v=\left\{s, \lambda_{1}, \ldots, \lambda_{r}\right\}$ such that the limit of the quotient $s / R$ is a fixed positive real number $\mu$, is the UIR of $I O(D-1)$ labeled by the parameter $\mu$ and the Young diagram $\lambda=\left\{\lambda_{1}, \ldots, \lambda_{r}\right\}$.

Proof: The use of the spherical harmonics construction discussed at the end of Subsection 4.4 is very convenient here. The main idea is to solve the homogeneity condition in a neighborhood of $x^{D} \neq 0$ as follows:

$$
\Phi\left(x^{m}, x^{D}, t_{\underline{a}}\right)=z^{s} \phi\left(\frac{x^{m}}{z}, t_{\underline{a}}\right),
$$

where $\vec{x}=\left(x^{m}, x^{D}\right)$ and $\phi\left(y^{m}, t_{\underline{a}}\right):=\Phi\left(y^{m}, \frac{s}{\mu}, t_{\underline{a}}\right)$. In other words, one may perform a convenient change of coordinates from the homogenous coordinates $\left(x^{m}, x^{D}\right)$ to the $\operatorname{set}\left(y^{m}, z\right)$ where

$$
y^{m}=\frac{x^{m}}{z}
$$

are the inhomogenous coordinates (on the projective space $\mathbb{P R}^{D-1}$ minus the point at infinity $z=0)$ and

$$
z=\frac{\mu x^{D}}{s}
$$

is a scale variable. The magic is that the equations for the generalized spherical harmonics have a well-behaved limit $x^{D} \rightarrow \infty$ in terms of $\phi\left(y^{m}, t_{a}\right)$ when $x^{D} / s$ is fixed to be equal to the ratio $z / \mu$, where $z$ and $\mu$ are finite [19]. To see that, one should use the relations

$$
\begin{aligned}
\frac{\partial}{\partial x^{m}} & =\frac{1}{z} \frac{\partial}{\partial y^{m}}, \\
\frac{\partial}{\partial x^{D}} & =\frac{\mu}{s}\left(\frac{\partial}{\partial z}-\frac{1}{z} y^{m} \frac{\partial}{\partial y^{m}}\right) .
\end{aligned}
$$

Moreover, the equations in this limit may be identified with equations for the proper UIR of the inhomogeneous orthogonal group $I O(D-1)$ realized homogeneously in terms of the inhomogenous coordinates. 
Example: The simplest instance is when $\lambda=\{0\}$ because one considers the sequence of harmonic functions $\Phi\left(x^{m}, x^{D}\right)$ of homogeneity degree $s$. The Laplace operator acting on $\Phi\left(x^{m}, x^{D}\right)$ reads in terms of $\phi\left(y^{m}\right)$ as follows

$$
\Delta_{\mathbb{R}^{D}} \Phi=z^{s-2}\left[\frac{\partial}{\partial y} \cdot \frac{\partial}{\partial y}+\frac{\mu^{2}}{s^{2}}\left(s(s-1)-(2 s-1)\left(y \cdot \frac{\partial}{\partial y}\right)+\left(y \cdot \frac{\partial}{\partial y}\right)^{2}\right)\right] \phi,
$$

due to the homogeneity condition (46) and the relations (47). The Laplace equation $\Delta_{\mathbb{R}^{D}} \Phi=0$ is thus equivalent to the equation

$$
\left[\frac{\partial}{\partial y} \cdot \frac{\partial}{\partial y}+\frac{\mu^{2}}{s^{2}}\left(s(s-1)-(2 s-1)\left(y \cdot \frac{\partial}{\partial y}\right)+\left(y \cdot \frac{\partial}{\partial y}\right)^{2}\right)\right] \phi=0,
$$

whose limit for $s \rightarrow \infty$ is the Helmholtz equation $\left[\Delta_{\mathbb{R}^{D-1}}+\mu^{2}\right] \phi=0$, where $\Delta_{\mathbb{R}^{D-1}}=\frac{\partial}{\partial y} \cdot \frac{\partial}{\partial y}$. The space of solutions of the Helmholtz equation carries an UIR of $I O(D-1)$ induced from a trivial representation of the stability subgroup $O(D-2)$.

\section{Relativistic field equations}

The Bargmann -Wigner programme amounts to associating, with any given UIR of the Poincaré group, a manifestly covariant differential equation whose (positive-energy) solutions transform according to the corresponding UIR. Physically, it might be natural to restrict this programme to the two most important classes of UIRs: the massive and massless representations. Mathematically, this restriction is convenient because the group-theoretical analysis is simpler since any of these UIRs is induced from an UIR of a unimodular orthogonal group $S O(n)$ (with $D-3 \leqslant n \leqslant D-1$ ), as can be checked easily on the tables of Subsection 3.3.

In 1948, this restricted programme was completed by Bargmann and Wigner in four dimensions when, for each such UIR of $I S O(3,1)^{\uparrow}$, a relativistic field equation was written whose positive-energy solutions transform according to the corresponding UIR [4]. But this case $(D=4)$ will not be reviewed here in details because it may cast shadow on the generic case. Indeed, it is rather peculiar in many respects:

- The quadratic and quartic Casimir operators essentially classify the UIRs, but this is no more true in higher dimensions where more Casimir operators are necessary and the classification quickly becomes technically cumbersome in this way. Moreover, one should stress that the eigenvalues of the Casimir operators do not characterize uniquely an irreducible representation (for instance, the quadratic and quartic Casimir operators vanish for all helicity representations).

- The (complex) Lorentz algebra $\mathfrak{s o}(3,1)$ is isomorphic to the direct sum of two (complex) rotation algebras $\mathfrak{s o}(3) \cong \mathfrak{s p}(2)$. These isomorphisms allow the use of the convenient "dotted-undotted" formalism for the finite-dimensional (non-unitary) irreps of the spin group $\operatorname{Spin}(3,1)$.

- The symmetric tensor-spinor fields are sufficient to cover all inequivalent cases.

- The helicity short little group $S O(2)$ is Abelian, therefore its irreps are one-dimensional, for fixed helicity. Notice that the helicity is discretized because the representation of the "little group" $S O(2)$ is a restriction of the representation of the group $\operatorname{Spin}(3) \cong S U(2)$ which has no intrinsically projective representations.

- The infinite-spin short little group $S O(1)$ is trivial, thus there are only two inequivalent infinite-spin representations (single- or double-valued) [6]. 
- etc.

Moreover, there exists an extensive literature on the subject of UIRs of $I S O(3,1)^{\uparrow}$ and we refer to the numerous pedagogical reviews available for more details on the four-dimensional case (see e.g. the inspiring presentations of [5] and [21]).

It is standard to require time reversal and parity symmetry of the field theory. More precisely, the field equations we will consider are covariant under the two previous transformations. As a consequence of the time reversal symmetry, the representation is irreducible under the group $\operatorname{ISO}(D-1,1)$ but reducible under the Poincaré group $\operatorname{ISO}(D-1,1)^{\uparrow}$ : the Hilbert space of solutions contain both positive and negative energy solutions. Furthermore, the parity symmetry implies that the representation is irreducible under the inhomogeneous Lorentz group $I O(D-1,1)$ but reducible under the group $I S O(D-1,1)$ (for instance, both chiralities are present in the massless case for $D$ even). To conclude, the Bargmann -Wigner programme is actually understood as associating, with any given UIR of the inhomogeneous Lorentz group, a manifestly covariant differential equation whose solutions transform according to the corresponding UIR.

\subsection{General procedure}

The lesson on induced representations that we learned from Wigner implies the following strategy:

1. Pick a unitary representation of the (short) little group.

2. Introduce a wave function on $\mathbb{R}^{D-1,1}$ taking values in some (possibly non-unitary) representation of the Lorentz group $O(D-1,1)$ the restriction of which to the (short) little group contains the representation of step 1 .

3. Write a system of linear covariant equations, differential in position space $x^{\mu}$ thus algebraic in momentum space $p_{v}$, for the wave function of step 2 . These equations may not be independent.

4. Fix the momentum and check in convenient coordinates that the field equations of step 3 put to zero all "unphysical" components of the wave function. More precisely, verify that its non-vanishing components carry the unitary representation of step 1.

Proof: The fact that the set of linear differential equations is taken to be manifestly covariant ensures that the Hilbert space of their solutions carries a (infinite-dimensional) representation of $I O(D-1,1)$. The fourth step determines the representation of the little group by which it is induced.

In the physics literature, the fourth step is referred to as "looking at the physical degrees of freedom." If the (possibly reducible) representation is proven to be unitary, then this property is summarized in a "no-ghost theorem."

The Klein-Gordon equation $\left(p^{2} \pm m^{2}\right) \Psi=0$ is always, either present in the system of covariant equations or a consequence thereof. Consequently, the Klein-Gordon equation will be assumed implicitly from now on in the step 3 . Therefore, the step 4 will be immediately performed in a proper Lorentz frame. (We refer the reader to the Subsection 3.2 for more details.)

The two completions [22] and [23-25] of the Bargmann-Wigner programme for finitecomponent representations in Minkowski spacetime of dimension $D>3$ are reviewed, respec- 
tively, in the appendix A and in the subsections 5.2-5.3 for single-valued UIRs of the Poincaré group. ${ }^{6}$

The tachyonic case ${ }^{7}$ is more briefly discussed in Subsection 5.4. The zero-momentum representations are not considered here since they essentially are the unitary irreducible representations of the de Sitter spacetime $d S_{D-1}$. The latter have been reviewed in [27].

The Bargmann -Wigner programme for fractional-spin fields in three spacetime dimensions has been completed in $[29,30]$. More generally, the exhaustive completion of the Bargmann Wigner programme (for all representations) in Minkowski spacetime of dimension $D=3$ is briefly summarised in Appendix B.

\subsection{Massive representations}

The Bargmann -Wigner programme is easy to complete for massive UIRs because the massive stability subgroup is the orthogonal group $O(D-1) \subset O(D-1,1)$. By going to a rest-frame, the time-like momentum vector takes the form $p^{\mu}=(m, \overrightarrow{0}) \neq 0$. The physical components of the field are thus carrying a tensorial irrep of the group $O(D-1)$ of orthogonal transformations in the spatial hyperplane $\mathbb{R}^{D-1}$ orthogonal to $p^{\mu}$. In other words, the linear field equations should remove all components including time-like directions. These unphysical components are responsible for the fact that the Fock space is not endowed with a positive-definite norm.

Step 1. From the sections 1.3 and 4, one knows that any unitary representation of the orthogonal group $O(D-1)$ is a sum of UIRs which are finite-dimensional and thus, equivalent to a tensorial representation. Let us consider the UIR of $O(D-1)$ labeled by the allowed Young diagram $\lambda=\left\{\lambda_{1}, \lambda_{2}, \ldots, \lambda_{r}\right\}$ (i.e. the sum of the lengths of its first two columns does not exceed $D-1$ ).

Step 2. The simplest ${ }^{8}$ way to perform the Bargmann-Wigner programme in the massive case is to choose a covariant wave function whose components carry the (finite-dimensional and non-unitary) tensorial irrep of the Lorentz group $O(D-1,1)$ labeled by the Young diagram $\lambda$. As explained in the subsection 4.4, a convenient way of realizing this is in terms of a wave function $\Phi\left(p, u_{a}\right)$ polynomial in the auxiliary commuting variables $u_{a}^{\mu}$ satisfying the irreducibility conditions (33)-(34).

Step 3. The massive Klein-Gordon equation

$$
\left(p^{2}+m^{2}\right) \Phi=0
$$

has to be supplemented with the transversality conditions

$$
\left(p \cdot \frac{\partial}{\partial u_{a}}\right) \Phi=0,
$$

of the wave function.

Step 4. Looking at a fixed-momentum mode in its corresponding rest-frame $p^{\mu}=(m, \overrightarrow{0})$ leads to the fact that the components of the wave function along the timelike momentum are set to zero by (49): $\Phi=\Phi\left(p, \vec{u}_{a}\right)$. In words, $\Phi$ does not depend on the time components $u_{a}^{0}, \forall a$. In this case, the conditions (33)-(34) read as irreducibility conditions under the orthogonal group $O(D-1)$.

Example: Massive symmetric representations with "spin" equal to $s$ correspond to Young diagrams $\lambda=\{s\}$ made of one row of length equal to the integer $s$. In four spacetime dimensions,

\footnotetext{
${ }^{6}$ Spinorial irreps may be adressed analogously by supplementing the system of differential equations with Diraclike equations and gamma-trace constraints (see e.g. $[19,26]$ for more details).

${ }^{7}$ The discussion presented in the section 5.4 was not published before, it directly derives from private conversations between X.B. and J. Mourad.

${ }^{8}$ There are other possible equivalent representations. In the case $D=4$, see Sec. 5.7 of [5], Eq. (5.7.33).
} 
this representation is precisely what is usually called a "massive spin-s field."' 9 The covariant wave function $\Phi(p, u)$ obeys to the irreducibility conditions (33)-(34) of the components

$$
\left(u \cdot \frac{\partial}{\partial u}-s\right) \Phi=0, \quad\left(\frac{\partial}{\partial u} \cdot \frac{\partial}{\partial u}\right) \Phi=0 .
$$

The wave function $\Phi$ is homogeneous of degree $s$ and harmonic in the auxiliary variable $u$. If the wave function $\Phi(p, u)$ is polynomial in the auxiliary variable $u$, then its components correspond to a symmetric tensor of rank $s$

$$
\Phi(p, u)=\frac{1}{s !} \Phi_{\mu_{1} \ldots \mu_{s}}(p) u^{\mu_{1}} \ldots u^{\mu_{s}},
$$

which is traceless

$$
\eta^{\mu_{1} \mu_{2}} \Phi_{\mu_{1} \mu_{2} \mu_{3} \ldots \mu_{s}}(p)=0
$$

The covariant field equations are the massive Klein-Gordon equation together with the transversality condition

$$
\left(p \cdot \frac{\partial}{\partial u}\right) \Phi=0
$$

which reads in components as

$$
p^{\mu_{1}} \Phi_{\mu_{1} \mu_{2} \ldots \mu_{s}}(p)=0 \text {. }
$$

The non-vanishing components of a solution of (53) must be along the spatial directions, i.e. only $\Phi_{i_{1} \ldots i_{s}}(p)$ may be $\neq 0$. This symmetric tensor field is traceless with respect to the spatial metric: $\delta^{i_{1} i_{2}} \Phi_{i_{1} i_{2} i_{3} \ldots i_{s}}(p)=0$, thus the physical components carry a symmetric irrep of the orthogonal group $O(D-1)$, the dimension of which can be computed by making use of the formula (25). The polynomial wave function $\Phi(p, u)$ evaluated on the internal unit hypersphere $u^{i} u_{i}=1$ corresponds to a decomposition of the physical components in terms of the spherical harmonics on the internal hypersphere $S^{D-2}$, which is an equivalent, though rather unusual, way of representing the physical components (usually, the use of spherical harmonics is reserved to the "orbital" part of the wave function).

The quartic Casimir operator of the Poincaré algebra is easily evaluated in components in the rest frame

$$
\begin{aligned}
-\frac{1}{2} \quad & P^{2} M_{\mu \nu} M^{\mu \nu}+M_{\mu \rho} P^{\rho} M^{\mu \sigma} P_{\sigma} \\
& =\frac{1}{2} m^{2}\left(M_{i j} M^{i j}+2 M_{i 0} M^{i 0}\right)-m^{2} M_{i 0} M^{i 0}=m^{2} \frac{1}{2} M_{i j} M^{i j},
\end{aligned}
$$

giving as a final result for a massive representation associated with a Young diagram $\lambda$

$$
\begin{aligned}
\mathcal{C}_{4}(\mathfrak{i s o}(D-1,1)) & =\mathcal{C}_{2}(\mathfrak{i s o}(D-1,1)) \mathcal{C}_{2}(\mathfrak{s o}(D-1)) \\
& =m^{2} \sum_{a=1}^{r} \lambda_{a}\left(\lambda_{a}+D-2 a-1\right)
\end{aligned}
$$

where the eigenvalues of the quadratic Casimir operator of the rotation algebra are given by the formula (27).

Example: In any dimension $D$, the eigenvalue of the quartic Casimir operator for a massive symmetric representation of rank $s$ is equal to $m^{2} s(s+D-3)$. In four spacetime dimensions, the square of the Pauli-Lubanski vector acting on a massive field of spin-s is indeed equal to $m^{2} s(s+1)$.

\footnotetext{
${ }^{9}$ To our knowledge, the Bargmann -Wigner programme for the massive integer-spin representations in fourdimensional Minkowski spacetime was adressed along the lines reviewed here for the first time by Fierz in [31].
} 
Each massive representation in $D \geqslant 4$ dimensions may actually be obtained as the first Kaluza-Klein mode in a dimensional reduction from $D+1$ down to $D$ dimensions. There is no loss of generality because the massive little group $S O(D-1)$ in $D$ dimension is identified with the $(D+1)$-dimensional helicity (short) little group. Such a Kaluza-Klein mechanism leads to a Stückelberg formulation of the massive field.

The massless limit $m \rightarrow 0$ of a massive irrep with $\lambda$ fixed is, in general, reducible because the irrep of the massive little group $S O(D-1)$ is restricted to the helicity (short) little group $S O(D-2) \subset S O(D-1)$. This argument combined with the known branching rule for $O(D-1) \downarrow O(D-2)$ (reviewed in Subsection 4.3) allows to prove that the massless limit of a massive irrep of the homogeneous Lorentz group labeled by a fixed Young diagram $\lambda$ contains each helicity irrep labeled by Young diagrams $\mu$ such that

$$
\lambda_{1} \geqslant \mu_{1} \geqslant \lambda_{2} \geqslant \mu_{2} \geqslant \ldots \geqslant \mu_{r-1} \geqslant \lambda_{r} \geqslant \mu_{r} \geqslant 0,
$$

with multiplicity one. The zero modes of a dimensional reduction from $D+1$ down to $D$ dimensions are determined by the same rule.

Example: The zero modes of the dimensional reduction of a massive symmetric representations with "spin" equal to $s$ are all helicity symmetric representations with integer "spins" not greater than the integer $s$, each with multiplicity one. For the dimensional reduction of a gravitational theory (i.e. a spin-two particle), one recovers the usual result that the massless spectrum is made of one "graviton" (spin-2), one "photon" (spin-1) and one "dilaton" (spin-0).

\subsection{Massless representations}

The quartic Casimir operator of the Poincaré algebra is evaluated easily in components in the light-cone coordinates (see Subsection 3.2 for notations),

$$
-\frac{1}{2} P^{2} M_{\mu \nu} M^{\mu \nu}+M_{\mu \rho} P^{\rho} M^{\mu \sigma} P_{\sigma}=0+M_{m+} P^{+} M^{m-} P_{-}=\pi_{m} \pi^{m},
$$

giving as a final result for a massless representation

$$
\mathcal{C}_{4}(\mathfrak{i s o}(D-1,1))=\mathcal{C}_{2}(\mathfrak{i s o}(D-2))=\mu^{2},
$$

where the quadratic Casimir operator of the massless little group is written in (17).

\subsubsection{Helicity representations}

Helicity representations correspond to the case $\mu=0$, so that $\pi^{m}=0$ and in practice the representation is induced from a representation of the orthogonal group $O(D-2)$.

Step 1. Again, any unitary representation of the orthogonal group $O(D-2)$ is a sum of finite-dimensional UIRs. Let us consider the UIR of the helicity short little group $O(D-2)$ labeled by the allowed Young diagram $\lambda=\left\{\lambda_{1}, \lambda_{2}, \ldots, \lambda_{r}\right\}$ (that is, the sum of the lengths of its first two columns does not exceed $D-2)$ :

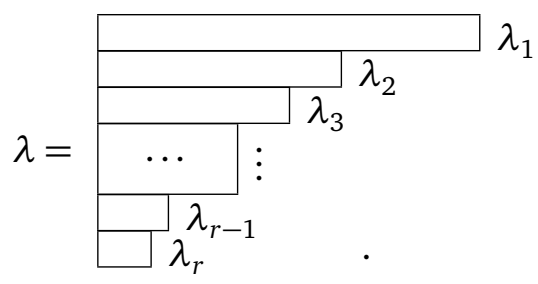

The step 2 is more subtle to perform than for massive representations because the field equations must set to zero all components along the light-cone of the covariant wave function, 
because they are unphysical. In other words, the covariant wave equations should remove two directions, and not only one like in the massive case. This fact implies that the transversality is not a sufficient condition any more, it must be supplemented either by other equations or by gauge symmetries asserting that one may quotient the solution space by pure gauge fields. In these lecture notes, one focuses on two gauge-invariant formulations which may be respectively referred to as "Bargmann -Wigner formulation" in terms of the field strength and "gauge-fixed formulation" in terms of the potential.

\section{Bargmann-Wigner equations}

The so-called "Bargmann -Wigner equations" were actually first written by Dirac [32] in four-dimensional Minkowski spacetime in spinorial form. Their name originates from their decisive use in the completion of the Bargmann -Wigner programme [4]. The generalization of the Bargmann -Wigner equations to any dimension was presented in [23-25] for tensorial irreps (reviewed here) and in [26] for spinorial irreps. The latter programme had previously been completed in [28] with different equations.

Step 2. Let $\bar{\lambda}=\left\{\lambda_{1}, \lambda_{1}, \lambda_{2}, \ldots, \lambda_{r}\right\}$ be the Young diagram depicted as

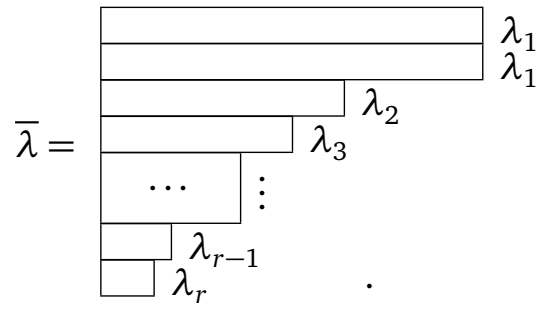

It is obtained from the Young diagram $\lambda$ represented in (56) by adding a row of equal length on top of the first row of $\lambda$. The Young diagram $\bar{\lambda}$ has at least two rows of equal lengths and the sum of the lengths of its first two columns does not exceed $D$. The covariant wave function is chosen to take values in the Schur module $V_{\bar{\lambda}}^{O(D-1,1)}$ realized in the manifestly antisymmetric convention. Following Subsection 4.4 , the wave function $\mathcal{K}\left(p, d_{I} x\right)$ is taken to be a polynomial in the graded variables $d_{I} x^{\mu}\left(I=1,2, \ldots, \lambda_{1}\right)$ obeying the commutation relations (30). Moreover, the irreducibility conditions of the components under the Lorentz group $O(D-1,1)$ are

$$
\left(d_{I} x^{\mu} \frac{\partial^{L}}{\partial\left(d_{J} x^{\mu}\right)}-\delta_{I J} \bar{\ell}_{I}\right) \mathcal{K}=0, \quad(I \leqslant J),
$$

where $\bar{\ell}_{I}$ stands for the length of the Ith column in the Young diagram $\bar{\lambda}$, and

$$
\left(\eta^{\mu \nu} \frac{\partial^{L}}{\partial\left(d_{I} x^{\mu}\right)} \frac{\partial^{L}}{\partial\left(d_{J} x^{\nu}\right)}\right) \mathcal{K}=0 .
$$

Step 3. The covariant field equations may be summarized in the assertion that the wave function is a "harmonic" multiform in the sense that, $\forall I$, it is "closed"

$$
\left(p_{\mu} d_{I} x^{\mu}\right) \mathcal{K}=0
$$

and "coclosed" (i.e. transverse)

$$
\left(p^{\mu} \frac{\partial^{L}}{\partial\left(d_{I} x^{\mu}\right)}\right) \mathcal{K}=0 .
$$

The operators $p \cdot d_{I} x$ act as "exterior differentials" (or "curls"), they are nilpotent and obey graded commutation relations. As one can easily see, the field equations (60) and (61), considered together, imply the massless Klein-Gordon equation. Actually, the equations (60) may even be imposed off-shell, whereas the equations (61) only hold on-shell [23-25]. 
Step 4. In the light-cone frame (see Section 1.1), the components of the momentum may be taken to be $p_{\mu}=\left(p_{-}, 0,0, \ldots, 0\right)$ with $p_{-} \neq 0$. On the one hand, the transversality condition (61) implies that the wave function does not depend on the variables $d_{I} x^{+}$. On the other hand, the closure condition (60) reads $\left(p_{-} d_{I} x^{-}\right) \mathcal{K}=0$, the general solution of which is $\mathcal{K}=\left(\prod_{I} p_{-} d_{I} x^{-}\right) \phi$, where $\phi$ depends neither on $d_{I} x^{-}$nor on $d_{I} x^{+}$(due to the transversality condition). In other words, the directions along the light-cone have been removed, since $\phi=\phi\left(p, d_{I} x^{m}\right)(m=1,2, \ldots, D-2)$. Focusing on this field, one may show that the irreducibility conditions (58) become, in terms of the function $\phi$,

$$
\left(d_{I} x^{m} \frac{\partial^{L}}{\partial\left(d_{J} x^{m}\right)}-\delta_{I J} \ell_{I}\right) \phi=0, \quad(I \leqslant J)
$$

where $\ell_{I}=\bar{\ell}_{I}-1$, and the trace conditions (59) implies

$$
\left(\delta^{m n} \frac{\partial^{L}}{\partial\left(d_{I} x^{m}\right)} \frac{\partial^{L}}{\partial\left(d_{J} x^{n}\right)}\right) \phi=0 .
$$

Since $\ell_{I}$ is the length of the $I$ th column of the Young diagram $\lambda$, the system of equations (62)(63) states that the components of the function $\phi$ carry a tensorial irrep of the orthogonal group $O(D-2)$. Therefore, the same is true for the physical components of the wave function $\mathcal{K}$.

This may be reformulated covariantly by saying that the closure (60) of the wave function implies that

$$
\mathcal{K}=\left(\prod_{I=1}^{\lambda_{1}} p_{\mu} d_{I} x^{\mu}\right) \phi .
$$

In components, this means that the tensor $\mathcal{K}$ is equal to $\lambda_{1}$ curls of the tensor $\phi$. This motivates the name "field strength" for the wave function $\mathcal{K}\left(p, d_{I} x\right)$, the components of which are irreducible under the Lorentz group (when evaluated on zero-mass shell) and labeled by $\bar{\lambda}$, and the name "potential" or "gauge field" for the wave function $\phi\left(p, d_{I} x\right)$, the components of which may be taken to be irreducible under the general linear group, with symmetries labeled by the Young diagram $\lambda$.

\section{Examples:}

- The helicity vectorial representation corresponds to a Young diagram $\lambda=\{1\}$ made of a single box. In four spacetime dimensions, this representation is precisely what is usually called a "vector gauge field". The Young diagram $\bar{\lambda}=\{1,1\}$ is a single column made of two boxes. The wave function in momentum space is given by

$$
\mathcal{K}=\frac{1}{2} \mathcal{K}_{\mu \nu}(p) d x^{\mu} d x^{\nu},
$$

which carries an irrep of $G L(D, \mathbb{R})$ : the antisymmetric rank-two representation. As one can see, the wave function actually is a differential two-form, the components of which transforming as an antisymmetric tensor of rank two. The field equations (60) and (61), respectively, read in components

$$
p_{\mu} \mathcal{K}_{\nu \rho}+p_{\nu} \mathcal{K}_{\rho \mu}+p_{\rho} \mathcal{K}_{\mu \nu}=0 \quad \text { (Bianchi identities) }
$$

and

$$
p^{\mu} \mathcal{K}_{\mu \nu}=0 \quad \text { (transversality conditions). }
$$

The differential two-form $\mathcal{K}$ is indeed harmonic (closed and coclosed). In physical terms, one says that the field strength $\mathcal{K}_{\mu \nu}$ obeys to the Maxwell equations. As usual, the Bianchi identities imply that the field strength derives from a potential: $\mathcal{K}_{\mu \nu}=p_{\mu} \phi_{\nu}-p_{\nu} \phi_{\mu}$. In the 
light-cone coordinates, the transversality implies that the components $\mathcal{K}_{+v}$ vanish, thus the only non-vanishing components are $\mathcal{K}_{-n}=p_{-} \phi_{n}$. Therefore the only physical components correspond to a $(D-2)$-vector in the hyperplane transverse to the light-cone.

- Helicity symmetric representations with "helicity" (or "spin") equal to $s$ correspond to Young diagrams $\lambda=\{s\}$ made of one row of length equal to the integer $s$. In four spacetime dimensions, this representation is precisely what is usually called a "massless spin-s field". The Young diagram $\bar{\lambda}=\{s, s\}$ is a rectangle made of two row of length equal to the integer $s$. The wave function is thus a polynomial in the auxiliary variables

$$
\mathcal{K}=\frac{1}{2^{s}} \mathcal{K}_{\mu_{1} v_{1}|\ldots| \mu_{s} v_{s}} d_{1} x^{\mu_{1}} d_{1} x^{\nu_{1}} \ldots d_{s} x^{\mu_{s}} d_{s} x^{v_{s}},
$$

satisfying the irreducibility equations (58)-(59) with $\ell_{I}=2, \forall I \in\{1, \ldots, s\}$. The tensor $\mathcal{K}$ is, by construction, antisymmetric in each of the $s$ sets of two indices

$$
\mathcal{K}_{\mu_{1} v_{1}|\ldots| \mu_{s} v_{s}}=-\mathcal{K}_{v_{1} \mu_{1}|\ldots| \mu_{s} v_{s}}=\ldots=-\mathcal{K}_{\mu_{1} v_{1}|\ldots| v_{s} \mu_{s}} .
$$

Moreover, the complete antisymmetrization over any set of three indices gives zero and all its traces are zero on-shell, so that the on-shell tensor $\mathcal{K}$ indeed belongs to the space irreducible under the Lorentz group $O(D-1,1)$ characterized by a two-row rectangular Young diagram of length $s$. In four-dimensional Minkowski spacetime, the irrep of the Lorentz group $O(3,1)$ carried by the on-shell tensor $\mathcal{K}$ is usually denoted as $(s, 0) \oplus(0, s)$. More precisely, the symmetry properties of the tensor $\mathcal{K}_{\mu_{1} v_{1}|\ldots| \mu_{s} v_{s}}$ are labeled by the Young tableau

$$
\begin{array}{|l|l|l|l|}
\hline \mu_{1} & \mu_{2} & \cdots & \mu_{s} \\
\hline v_{1} & v_{2} & \cdots & v_{s} \\
\hline
\end{array}
$$

The equation (64) means that the components of the tensor $\mathcal{K}_{\mu_{1} v_{1}|\ldots| \mu_{s} v_{s}}$ are essentially the projection of $p_{\mu_{1}} \ldots p_{\mu_{s}} \phi_{v_{1} \ldots v_{s}}$ on the tensor field irreducible under $G L(D, \mathbb{R})$ with symmetries labeled by the above Young tableau. The physical components $\phi_{n_{1} \ldots n_{s}}$ of the symmetric tensor gauge potential $\phi_{v_{1} \ldots v_{s}}$ are along the $D-2$ directions transverse to the light-cone. The number of physical degrees of freedom of a helicity symmetric field of rank $s$ can be computed by making use of the formula (25).

- The helicity symmetric representation with "spin" equal to 2 corresponds to the graviton. The field strength has the symmetry properties of the Riemann tensor. Its on-shell tracelessness indicates that it corresponds to the (linearized) Weyl tensor. The equations (60) are the Bianchi identities for the linearized Riemann tensor in flat spacetime, whereas the equations (61) hold as a consequence of the sourceless Einstein equations linearized around flat spacetime.

Remark: One can find some early indications for the existence of the tensor $\mathcal{K}^{\mu_{1} \nu_{1}|\ldots| \mu_{s} \nu_{s}}$ in the paper [33] where Weinberg constructs free quantum field operators that have a nonzero expectation value between the vacuum and one-particule states for massless particles of helicity $\pm s$ in four spacetime dimensions. In Weinberg's approach, one cannot find the classical (or "first-quantized") field strength tensor $\mathcal{K}^{\mu_{1} v_{1}|\ldots| \mu_{s} v_{s}}$ that we have built above, but instead a quantum operator (in so-called "second-quantization") that we denote here $\widehat{\mathcal{K}}_{ \pm} \mu_{1} v_{1}|\ldots| \mu_{s} v_{s}$ and that transforms like a tensor under Lorentz transformations. This operator is built out of the product $\left[p^{\mu_{1}} e_{ \pm}^{v_{1}}(\vec{p})-p^{v_{1}} e_{ \pm}^{\mu_{1}}(\vec{p})\right] \ldots\left[p^{\mu_{s}} e_{ \pm}^{v_{s}}(\vec{p})-p^{v_{s}} e_{ \pm}^{\mu_{s}}(\vec{p})\right]$ featuring the two polarisation "vectors" $e_{ \pm}^{\mu}(\vec{p})$. On the one hand, solving the Bianchi identities for the field strength $\mathcal{K}^{\mu_{1} v_{1}|\ldots| \mu_{s} v_{s}}$ allows to write the latter as an expression involving $s$ derivatives of a completely symmetric gauge potential $\phi_{\mu_{1} \ldots \mu_{s}}$. This potential satisfies [23-25] the second-order Fronsdal field equations [34] and is the building block for the construction of an interacting quantum field theory with long-range interactions. On the other hand, the canonical quantization of the free field theory with field strength tensor $\mathcal{K}$ gives rise to Weinberg's quantum field operator 
$\widehat{\mathcal{K}}_{ \pm}$. The same remarks apply to the relation between the generalised field strength (64) and its second-quantized version in [35].

Gauge-fixed equations

The following equations are somewhat unusual, but they proved to be crucial in the completion of the Bargmann -Wigner programme for the infinite spin representations [19].

Step 2. Let $\widehat{\lambda}=\left\{\lambda_{1}-1, \lambda_{2}-1, \ldots, \lambda_{r}-1\right\}$ be the Young diagram depicted as

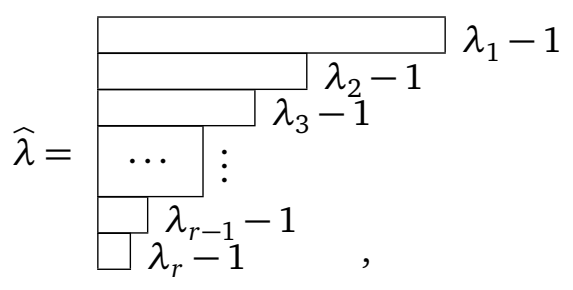

obtained from the Young diagram $\lambda$ represented in (56) by removing the first column of $\lambda$. Therefore the sum of the length of the first two columns of the Young diagram $\widehat{\lambda}$ does not exceed $D-2$. The covariant wave function is chosen to take values in the Schur module $V_{\widehat{\lambda}}^{O(D-1,1)}$ realized in the manifestly symmetric convention. Actually, as anticipated in Subsection 4.4, it turns out to be crucial to regard the wave function $\Phi\left(p, u_{a}\right)$ as a distribution in the commuting auxiliary variables $u_{a}^{\mu}$, obeying to

$$
\begin{gathered}
{\left[\left(u_{a} \cdot \frac{\partial}{\partial u_{b}}\right)-\widehat{\lambda}_{a} \delta_{a b}\right] \Phi=0, \quad(a \leqslant b),} \\
\left(\frac{\partial}{\partial u_{a}} \cdot \frac{\partial}{\partial u_{b}}\right) \Phi=0 .
\end{gathered}
$$

Step 3. Proper field equations are the transversality condition (49) combined with the equation

$$
\left(p \cdot u_{a}\right) \Phi=0 .
$$

The equations (69) and (49) are the respective analogues of the closure and coclosure conditions (60)-(61). A drastic difference is that the operators $p \cdot u_{a}$ are not nilpotent (thus there is no underlying cohomology). Actually, the equation (69) has no solution if $\Phi$ is assumed to be a polynomial in all the variables.

Step 4. Equation (69) can be solved as

$$
\Phi=\delta\left(u_{a} \cdot p\right) \phi
$$

where the distribution $\phi\left(p, u_{a}\right)$ may actually be assumed to be a function depending polynomially on the auxiliary variables $u_{a}$ for the present purpose. The Dirac delta is a distribution of homogeneity degree equal to minus one, hence the irreducibility conditions (67)-(68) imply that

$$
\begin{aligned}
{\left[\left(u_{a} \cdot \frac{\partial}{\partial u_{b}}\right)-\lambda_{a} \delta_{a b}\right] \phi } & =0 \quad(a \leqslant b), \\
\left(\frac{\partial}{\partial u_{a}} \cdot \frac{\partial}{\partial u_{b}}\right) \phi & =0 .
\end{aligned}
$$

The function $\phi$ is defined from (70) modulo the equivalence relation

$$
\phi \sim \phi+\sum_{a=1}^{r}\left(u_{a} \cdot p\right) \epsilon_{a},
$$


where $\epsilon_{a}$ are arbitrary functions. This means that (70) is equivalent to the alternative road towards the Bargmann -Wigner programme: the gauge symmetry principle with the irreducible components of $\left(u_{a} \cdot p\right) \epsilon_{a}$ being pure gauge fields. As mentioned before, this path will not be addressed here (see e.g. [23-25] and refs therein for more discussions on the gauge-invariance issue). Therefore, one may say that the equation (69) is the "remnant" of the gauge symmetries (73). In the light-cone coordinates, the gauge symmetries (73) imply that one may choose a representative $\phi$ which does not depend on the variables $u_{a}^{-}$(the gauge is "fixed"). The transversality condition (49) implies that $\phi$ is also transverse, implying no dependence on $u_{a}^{+}$ ("gauge shoots twice"). Thus $\phi$ depends only on the transverse auxiliary variables $u_{a}^{m}$, so one concludes by observing that the physical components of $\phi$ carry a tensorial irrep of $O(D-2)$ labeled by $\lambda$.

\subsubsection{Infinite spin representations}

Infinite spin representations correspond to the case $\mu \neq 0$ and, in practice, the representation of the massless little group $I O(D-2)$ is induced from a representation of the orthogonal group $O(D-3)$. The parameter $\mu$ is a real parameter with the dimension of a mass. Wigner proposed a set of manifestly covariant equations to describe fields carrying these UIR in four spacetime dimensions [36]. They have been generalized to arbitrary infinite-spin representations in any dimension [19]. ${ }^{10}$

Step 1. Again, any unitary representation of the orthogonal group $O(D-3)$ is a sum of finite-dimensional UIRs. Let us consider the UIR of the helicity short little group $O(D-3)$ labeled by the allowed Young diagram $\lambda=\left\{\lambda_{1}, \lambda_{2}, \ldots, \lambda_{r}\right\}$ (that is, the sum of the lengths of its first two columns does not exceed $D-3)$.

Step 2. In order to have manifest covariance, it is necessary to lift the eigenvalues $\xi^{m}$ of the generators $\pi^{m}$ in the massless little group to a $D$-vector $\xi^{\mu}$. In practice, the covariant wave function is taken to be a distribution $\Phi\left(p, \xi, u_{a}\right)$ satisfying the conditions (33)-(34). The tensorial components associated with the commuting variables $u_{a}$ belong to the Schur module of the Lorentz group $O(D-1,1)$ labeled by an allowed Young diagram $\lambda$.

Step 3. Relativistic equations describing a first-quantized particle with infinite spin are

$$
\begin{aligned}
(p \cdot \xi) \Phi & =0, \\
\left(p \cdot \frac{\partial}{\partial \xi}-i\right) \Phi & =0, \\
\left(\xi^{2}-\mu^{2}\right) \Phi & =0,
\end{aligned}
$$

together with the transversality conditions

$$
\begin{aligned}
\left(p \cdot u_{a}\right) \Phi & =0, \\
\left(p \cdot \frac{\partial}{\partial u_{a}}\right) \Phi & =0, \\
\left(\xi \cdot \frac{\partial}{\partial u_{a}}\right) \Phi & =0 .
\end{aligned}
$$

This system of equations is far from being independent. For instance, compatibility condition of the systems (74)-(75) or (77)-(78) is the massless Klein-Gordon equation.

Step 4. The equation (75) reflects the fact that the couples $(p, \xi)$ and $(p, \xi+\alpha p)$ are physically equivalent for arbitrary $\alpha \in \mathbb{R}$. Indeed, one gets

$$
\Phi(p, \xi+\alpha p)=e^{i \alpha} \Phi(p, \xi)
$$

\footnotetext{
${ }^{10}$ More recent developments (as well as a list of open challenges) have been reviewed in [20].
} 
from Equation (75). The equation (76) states that the internal vector $\xi$ is a space-like vector while the mass-shell condition states that the momentum is light-like. From the equation (74), one obtains that the internal vector is transverse to the momentum. All together, one finds that $\xi$ may be taken to live on the hypersphere $S^{D-3}$ of radius $\mu$ embedded in the transverse hyperplane $\mathbb{R}^{D-2}$. In brief, the "continuous spin" degrees of freedom essentially correspond to $D-3$ angular variables, whose Fourier conjugates are discrete variables analogous to the usual spin degrees of freedom. Finally, proceeding analogously to the "gauge-fixed" field equations of the helicity representations, one may show [19] that the conditions (77)-(79) concretely remove three unphysical directions in the components, so that the final result is a tensorial irrep of the short little group $O(D-3)$ fixing both the momentum $p$ and the internal vector $\xi$.

From the group theoretical point of view, the UIR of the homogeneous and inhomogeneous orthogonal groups are related by an Inönü-Wigner contraction $O(D-1) \rightarrow I O(D-2)$ (see Subsection 4.5). It follows that one can obtain the continuous spin representations from the massive ones in a suitable massless limit $m \rightarrow 0$ since their little group UIRs are related by a contraction. The quartic Casimir operator of the Poincaré group for the massive representation is related to its Young diagram $v$ labeling the UIR of the little group $O(D-1)$ via the formula (54):

$$
\mathcal{C}_{4}(\mathfrak{i s o}(D-1,1))=m^{2} \sum_{a=1}^{r} v_{a}\left(v_{a}+D-2 a-1\right) .
$$

In order to keep $\mathcal{C}_{4}$ non-vanishing, the massless limit must be such that the product of the "spin" $v_{1}=s$ and the mass $m$ remains finite. More precisely, one needs $s m \rightarrow \mu$ in order to reproduce (55), so that the spin goes to infinity while the row lengths $v_{a}$ for $a \neq 1$ are kept equal to $\lambda_{a-1}[19,37]$. The Fourier transform (in the internal space spanned by $\xi$ ) of the field equations (74)-(79) may be obtained in this way from the field equations of a massive representation in "gauge-fixed" form (see [19] for more details). This limit is very similar to the contraction of Subsection 4.5.

\subsection{Tachyonic representations}

The tachyonic representations have some similarities with the massive representations. The simpler one is the analogue of the Klein-Gordon equation, up to a change of sign for the mass term. The other similarity is that the linear equations should remove the components along the momentum. Of course, the major difference is that the momentum is space-like. The quartic Casimir operator of the Poincaré algebra is also evaluated easily in components, giving as a final result for a tachyonic representation,

$$
\mathcal{C}_{4}(\mathfrak{i s o}(D-1,1))=\mathcal{C}_{2}(\mathfrak{i s o}(D-1,1)) \mathcal{C}_{2}(\mathfrak{s o}(D-2,1)),
$$

where the eigenvalues of the quadratic Casimir operator of the rotation algebra are given by the formula (27).

Step 1. The first step is more involved for the tachyonic case since it requires the exhaustive knowledge of the UIR theory for the groups $S O(D-2,1)^{\uparrow}$. Fortunately, complete results are available [38-41]. The steps 2-3 further require the completion of the Bargmann -Wigner programme for the isometry group $S O(D-2,1)^{\uparrow}$ of the de Sitter spacetime $d S_{D-2}$. This has been done in [27]. ${ }^{11}$

Let us assume that this programme has been performed through an ambient space formulation, analogous to the one of the spherical harmonics, as discussed in the subsection 4.4. More explicitly, let us consider that the physical components of the wave function have been

\footnotetext{
${ }^{11}$ The Bargmann -Wigner programme in $A d S_{D}$, with field equations that generalise the ones presented in Section 5.3 , were obtained in $[42,43]$. Similar equations were obtained later in the $d S_{D}$ signature [27].
} 
realized via a function on the hyperboloid $d S_{D-2}$ of radius $\mu>0$ embedded in $\mathbb{R}^{D-2,1}$ with some set of auxiliary commuting vectors of $\mathbb{R}^{D-2,1}$ (for the spin degrees of freedom) and the corresponding $O(D-2,1)$-covariant field equations of the UIR are known explicitly. The step 1 is therefore assumed to be performed.

Step 2. In order to have manifest Lorentz invariance, all auxiliary variables are lifted to $D$-vectors: the coordinates of the internal de Sitter spacetime are denoted by $\xi^{\mu}$ and the auxiliary variables by $u_{A}^{\mu}$. The wave function is taken to be $\Phi\left(p, \xi, u_{A}\right)$, where the internal vector $\xi$ plays a role similar to the one in the infinite-spin representations. An important distinction is that in the ambient space formulation, one would evaluate the wave function on the hypersurface $\xi^{2}=\mu^{2}$ instead of imposing this relation on the wave function, as in (76). The $O(D-2,1)$-covariant field equations for the UIR of the little group $O(D-2,1)$ must be $O(D-1,1)$-covariantized accordingly. Concretely, this implies that the components of the covariant wave function carry an (infinite-dimensional) irrep of the Lorentz group.

Step 3. These covariantized field equations and the tachyonic Klein-Gordon equation $\left(p^{2}-m^{2}\right) \psi=0$ must be supplemented by two equations: say the orthogonality condition (74), similarly to the infinite spin representation, and the transversality condition (49), similarly to the massive representation. The orthogonality condition (74) may be replaced by another transversality equation for the vector $\xi$.

Step 4. Now, the equation (74) implies that the internal vector belongs to the hyperplane $\mathbb{R}^{D-2,1}$ orthogonal to the momentum $p$. Its intersection with the hypersurface $\xi^{2}=\mu^{2}$ restricts $\xi$ to the internal de Sitter space $d S_{D-2} \subset \mathbb{R}^{D-2,1}$. Moreover, the condition (49) sets to zero all components of the wave function along the momentum. Therefore, the remaining components are physical and carry an UIR of the little group $O(D-2,1)$ by construction (see step 2 ).

Example: The simplest non-trivial example corresponds to a tachyonic representation of the inhomogeneous Lorentz group $I O(D-1,1)$ induced by a representation of the little group $O(D-2,1)$ corresponding to "massive scalar field" on the "internal de Sitter spacetime" $d S_{D-2}$ with $D \geqslant 4$. This UIR belongs to the principal continuous series of UIR of the group $O(D-2,1)$ and it may be realized as the space of harmonic functions on $\mathbb{R}^{D-2,1}$ of (complex) homogeneity degree $s$ equal to $\frac{3-D}{2}+i \sigma$ (with $\sigma$ a positive real parameter) evaluated on the unit onesheeted hyperboloid $d S_{D-2} \subset \mathbb{R}^{D-2,1}$. They can be regarded as a generalization of the spherical harmonics in the Lorentzian case, where the degree is a complex number. The eigenvalue of the quadratic Casimir operator (4) of the little group $O(D-2,1)$ on this representation is equal to

$$
\mathcal{C}_{2}(\mathfrak{s o}(D-2,1))=\left(\frac{D-3}{2}\right)^{2}+\sigma^{2} .
$$

The d'Alembertian on the unit hyperboloid evaluated on such functions is precisely equal to the former eigenvalue (as is true for the Laplacian on the unit sphere evaluated on spherical harmonics) so the corresponding fields on the internal spacetime $d S_{D-2}$ are indeed "massive". Inserting the above result in (82), one sees that the quartic Casimir operator is negative for the corresponding tachyonic representation. In four-dimensional Minkowski spacetime, this implies that the Pauli-Lubanski vector is time-like. The Lorentz-covariant wave function is taken to be $\Phi(p, \xi)$ evaluated on $\xi^{2}=1$ and the corresponding relativistic equations for the 
induced tachyonic representation may be chosen as

$$
\begin{aligned}
\left(p^{2}-m^{2}\right) \Phi & =0, \\
\left(p \cdot \frac{\partial}{\partial \xi}\right) \Phi & =0, \\
\left(\frac{\partial}{\partial \xi} \cdot \frac{\partial}{\partial \xi}\right) \Phi & =0, \\
\left(\xi \cdot \frac{\partial}{\partial \xi}-s\right) \Phi & =0,
\end{aligned}
$$

where one should remember that $s=\frac{3-D}{2}+i \sigma$. Notice the formal analogy with the system of equations (48), (52)) and (50) for a massive symmetric tensor field.

Remark: There might be sometimes confusion in the folklore surrounding the tachyons. We would like to insist on the fact that the tachyonic representations are indeed unitary (by definition). Still, their physical interpretation is problematic because they are not causal in the sense that one may show that the support of their propagator requires superluminal propagation. Roughly speaking, the acausality is obvious because the momentum is space-like, $p^{2}=+m^{2}$. The confusing point is that one may try to circumvent this problem in the following way: solving $p^{2}-m^{2}=0$ by $p^{\mu}=(i m, \overrightarrow{0})$ enforces causality, but the price to pay is the loss of unitarity. Indeed, the energy is pure imaginary, hence a naive plane-wave $e^{ \pm i p_{0} x^{0}}$ is actually a non-integrable exponential $e^{ \pm m x^{0}}$. These remarks are summarized in the following table:

\begin{tabular}{|c|c|c|c|}
\hline$E=p_{0}$ & $|\vec{p}|$ & Unitarity & Causality \\
\hline 0 & $m$ & OK & KO \\
$\pm i m$ & 0 & KO & OK \\
\hline
\end{tabular}

Nevertheless, the tachyonic representations should not be discarded too quickly on such physical grounds. Actually, if tachyonic representations appear in the spectrum of a theory, then it merely signals a local instability of the field theory in the sense that the perturbation theory is performed around an unstable vacuum, and the tachyon might roll to a stable vacuum (if any). For instance, the Higgs particle is described by nothing but a tachyonic scalar field (induced by the trivial representation of the little group). By analogy, one may wonder if some infinite-component tachyonic field (induced by a non-trivial representation of the little group) could not play a role in some huge Brout-Englert-Higgs mechanism providing mass to an infinite tower of gauge fields in various massless irreps.

\section{Acknowledgements}

We thank all the colleagues with whom we have worked and discussed on the Bargmann Wigner programme in maximally symmetric spacetimes. The authors also acknowledge the Institut des Hautes Études Scientifiques (Bures-sur-Yvette, France) and the Université de MonsHainaut (Mons, Belgium) for hospitality during the first stage of this work. The work of NB was partially supported by an F.R.S.-FNRS PDR grant "Fundamental issues in extended gravity" No T.0022.19.

\section{A Siegel-Zwiebach equations}

The Bargmann -Wigner programme for finite-component representations in Minkowski spacetime of any dimension $D>3$ was completed for massless helicity representations by Siegel 
and Zwiebach in [28] and generalised to massive representations in Siegel's lecture notes [22]. Only the massless representations will be reviewed here since the case of massive representations follows by dimensional reduction, as mentioned in the subsection 5.2.

\section{Siegel-Zwiebach equations}

The main idea behind these equations is the covariantisation of the condition that the "translation" generators $\pi_{n}$ of the massless little group $I O(D-2)$ must act trivially on physical states of the helicity representations (cf. Subsections 3.2-3.3). Let us rewind the procedure initiated in Subsection 5.3.1:

Steps 1 and 2. These first steps are identical to the case of Bargmann -Wigner equations, i.e. the wave function is a field strength $\mathcal{K}\left(p, d_{I} x\right)$ taking values in an irrep of the Lorentz group $O(D-1,1)$ labeled by the Young diagram $\bar{\lambda}$.

Step 3. The generators of the Lorentz algebra $\mathfrak{s o}(D-1,1)$ can be decomposed as the sum $M_{\mu \nu}=L_{\mu \nu}+S_{\mu \nu}$ of the "orbital" part (transforming the positions or momenta) and the "spin" part (transforming the irrep labeled by the Young diagram $\bar{\lambda}$ ),

$$
L_{\mu \nu}=-i\left(p_{\mu} \frac{\partial}{\partial p^{v}}-p_{v} \frac{\partial}{\partial p^{\mu}}\right), \quad S_{\mu \nu}=-i\left(d_{I} x_{\mu} \frac{\partial}{\partial\left(d x_{I}^{v}\right)}-d_{I} x_{v} \frac{\partial}{\partial\left(d x_{I}^{\mu}\right)}\right) .
$$

The Siegel-Zwiebach equations for $s \neq 0$ take the simple form

$$
\left(p^{\mu} S_{\mu v}-i s p_{v}\right) \mathcal{K}=0 \text {. }
$$

They imply the massless Klein-Gordon equation $p^{2} \mathcal{K}=0$ (since $s \neq 0$ ). In fact, one can check that the quadratic and quartic Casimir operators both vanish as a consequence of (89). ${ }^{12}$ Notice that a similar "spin-enslaving" relation, leading to (89), was recently given in [44].

Step 4. In the light-cone frame (see Section 1.1) where the components of the momentum are $p_{\mu}=\left(p_{-}, 0,0, \ldots, 0\right)$ with $p_{-} \neq 0$, the system (89) of equations splits as

$$
\pi_{n} \mathcal{K}=0, \quad\left(S_{+-}-i s\right) \mathcal{K}=0,
$$

where $\pi_{n}:=p_{-} S_{+n}=p^{\mu} S_{\mu n}$ (with $n=1,2, \ldots, D-2$ ) are generators corresponding the "translation" subgroup $\mathbb{R}^{D-2} \subset I O(D-2)$ of the massless little group. ${ }^{13}$ On the one hand, the fact that these generators $\pi_{n}$ act trivially ensures that the massless representation is a helicity representation, i.e. only the generators $S_{m n}$ of the rotations in the transverse plane act nontrivially. Moreover, the condition $\pi_{n} \mathcal{K}=0$ implies that the field strength $\mathcal{K}$ in the light-cone frame has a maximal (respectively, minimal) number of factors $d_{I} x^{-}$(respectively, $\left.d_{I} x^{+}\right){ }^{14}$ Therefore, the physical components of the field strength read $\mathcal{K}=\left(\prod_{I} p_{-} d_{I} x^{-}\right) \phi$, where $\phi$ depends neither on $d_{I} x^{-}$nor on $d_{I} x^{+}$. On the other hand, the eigenvalue $S_{+-}=i s$ of the Lorentz generator

$$
S_{+-}=-i\left(d_{I} x^{+} \frac{\partial}{\partial\left(d_{I} x^{+}\right)}-d_{I} x^{-} \frac{\partial}{\partial\left(d_{I} x^{-}\right)}\right)
$$

implies that the Young diagram $\bar{\lambda}$ must have $s$ columns. This is because the operator $S_{+-}$is a number operator (up to a coefficient $i$ ) for the total number of covariant indices - minus the number of covariant indices + , and in every column of the field strength there is no index + and one index - . The conclusion that is reached is the same as in Subsection 5.3.1.

\footnotetext{
${ }^{12}$ In order to check that the quartic Casmir operator acts trivially, it useful to notice that $M_{\mu \nu}$ can be replaced everywhere by $S_{\mu \nu}$ inside the definition (6). In $D=4$, this property is obvious in terms of the Pauli-Lubanski vector.

${ }^{13}$ See Subsection 3.2. Note that $M_{+n}=S_{+n}$ and $M_{m n}=S_{m n}$ in this light-cone frame, since the corresponding orbital parts of the generators of the little group act trivially on the momentum.

${ }^{14}$ See [22] for an elegant derivation of these facts from (90).
} 


\section{Equivalence with Bargmann-Wigner equations}

In fact, the Siegel -Zwiebach equations are equivalent to the Bargmann -Wigner equations reviewed in Subsection 5.3.1. For instance, the closure and coclosure conditions (60) and (61) imply (89). This follows from the identity

$$
\begin{aligned}
p^{\mu} S_{\mu \nu} & =-i p^{\mu}\left(d_{I} x_{\mu} \frac{\partial}{\partial\left(d x_{I}^{v}\right)}-d_{I} x_{v} \frac{\partial}{\partial\left(d x_{I}^{\mu}\right)}\right) \\
& =-i\left(p_{\mu} d_{I} x^{\mu}\right) \frac{\partial}{\partial\left(d x_{I}^{v}\right)}-d_{I} x_{\nu}\left(p^{\mu} \frac{\partial}{\partial\left(d x_{I}^{\mu}\right)}\right) .
\end{aligned}
$$

In the last term, one recognises between the parentheses the divergence operator acting on the column $I$, which gives zero because of the coclosure condition (61). As for the first term on the right-hand side of the above equation, one can rewrite it as

$$
-i\left(p_{\mu} d_{I} x^{\mu}\right) \frac{\partial}{\partial\left(d x_{I}^{\nu}\right)}=-i \frac{\partial}{\partial\left(d x_{I}^{\nu}\right)}\left(p_{\mu} d_{I} x^{\mu}\right)-i p_{\mu}\left[d x_{I}^{\mu}, \frac{\partial}{\partial\left(d x_{I}^{\nu}\right)}\right] .
$$

The first term on the right-hand side gives zero on the field strength because of the closure relation (60), while the last term gives $+i s p_{v}$ because of the commutation relations $\left[d x_{I}^{\mu}, \frac{\partial}{\partial\left(d x_{I}^{v}\right)}\right]=-s \delta_{v}^{\mu}$.

The covariant proof that the Siegel-Zwiebach equations imply Bargmann -Wigner equations is more cumbersome and will not be presented here. Anyway, this equivalence is guaranteed from the light-cone frame analysis.

\section{B Bargmann -Wigner programme in three dimensions}

In this appendix we review results obtained in the literature concerning the Wigner and Bargmann-Wigner programmes in Minkowski spacetime of dimension $D=2+1$. The former programme was achieved in [10] along the lines of the seminal paper [6] by Wigner.

There are four classes of UIRs of the Poincaré group $\operatorname{ISO}(2,1)^{\uparrow}$ :

1) Zero-momentum representations, labeled by the eigenvalue $c \in \mathbb{R}$ of the quadratic Casimir operator $\mathcal{C}_{2}[\mathfrak{s o}(2,1)]$ of the Lorentz algebra $;{ }^{15}$

2) Massive representations, labeled by mass $m>0$ and spin $s \in \mathbb{R}$;

3) Massless representations:

1. helicity representations, either single-valued (bosonic) or double-valued (fermionic);

2. infinite-spin representations, labeled by a dimensionful parameter $\mu>0$;

4) Tachyonic representations, labeled by a dimensionful parameter $m>0$ and by a dimensionless parameter $s \in \mathbb{R}$ (the analogue of spin).

In what follows, we briefly summarize exhaustive results on the completion of the Bargmann Wigner programme in $D=2+1$ dimensions for the four classes of UIRs listed above.

\footnotetext{
${ }^{15}$ Strictly speaking, the principal and complementary series are labeled by two real parameters, not only by the value of the Casimir operator.
} 


\section{B.1 Zero momentum representations}

Effectively, the zero momentum representation of the Poincare group $\operatorname{ISO}(2,1)^{\uparrow}$ are UIRs of the Lorentz subgroup $S O(2,1)^{\uparrow}$. The latter were classified in [38]. We also refer the reader to [45] for a physicist-friendly classification of the irreps of the Lorentz group $S O(2,1)^{\uparrow}$.

We will not repeat these well-known results here. For the purpose of the Bargmann -Wigner programme, it is enough to know that the UIRs of $S O(2,1)^{\uparrow}$ are labeled by the real eigenvalue of the quadratic Casimir operator $\mathcal{C}_{2}[\mathfrak{s o}(2,1)]$ of the Lorentz algebra (and another real parameter for the principal and complementary series, cf. Footnote 15). Since the momentum is vanishing, the states span a constant field $\psi$ on Minkowski spacetime taking values in these UIRs of the Lorentz group $S O(2,1)^{\uparrow}$. A relativistic equation is then $\left(\mathcal{C}_{2}[\mathfrak{s o}(2,1)]-c\right) \psi=0$, which asserts that the states $\psi$ are eigenvectors of the Casimir operator with eigenvalue $c \in \mathbb{R}$.

\section{B.2 Massive representations}

Consider a massive representation labeled by mass $m>0$ and spin $s \in \mathbb{R}$.

\section{B.2.1 (Half-)integer spins}

For integer spin $s \in \mathbb{N}$, the Klein-Gordon equation (48) together with the tracelessness condition (51) and the transversality condition (53) for a totally symmetric tensor $\varphi_{\mu_{1} \ldots \mu_{s}}$ provide relativistic field equations whose positive-energy solutions represent the corresponding UIR. Equivalently, for non-vanishing integer spin $s \in \mathbb{N}_{0}$, they can be summarized by the following set of equations:

$$
\eta^{\mu_{1} \mu_{2}} \varphi_{\mu_{1} \ldots \mu_{s}}=0, \quad m \varphi_{\mu_{1} \ldots \mu_{s}} \pm i \epsilon_{\mu_{1} v \rho} p^{v} \varphi^{\rho}{ }_{\mu_{2} \ldots \mu_{s}}=0,
$$

where we take $\epsilon_{012}=-1$. For early references, see $[29,30,46,47]$. These equations are explicitly written in [48] and can be found in spinor notation in [49]. Notice that the transversality condition (53) directly follows from the second equation in (94). Moreover, note that there is no need to explicitly symmetrize the last equation in its free indices when the tracelessness and transversality conditions hold true. In turn, the Klein-Gordon equation follows from repeated application of the second equation in (94). The two possible signs in the last equation stand for the two possible values $\pm s$ of the "helicity" of the massive particle. This system of equations can be generalized to $\mathrm{AdS}_{3}$; see [50] for a classification.

\section{B.2.2 Fractional spins}

In the case of the massive UIRs where the real number $s$ is neither integer nor half-integer ("fractional spin", see e.g. [51] for a review), one should stress that although the number of physical components is one (the UIRs of the massive little group $S O(2)$ are one-dimensional since this group is Abelian) nevertheless their corresponding covariant description necessarily involve relativistic field equations with an infinite number of components (since there are no finite-dimensional irreps of the Lorentz group $S O(2,1)^{\uparrow}$ with such values of the spin).

The positive-energy solutions to the system of the four equations (48), (50), (51), (52) formally describe a massive UIR of mass $m$ and spin $s \in \mathbb{R}$ (as can be checked by computing the value of the quartic Casimir operator). Note that the field $\Phi(p, u)$ is not polynomial in the auxilliary vector $u^{\mu}$ when $s \notin \mathbb{N}$. Finding a suitable functional space is a subtle issue that we will not attempt to address. In fact, the construction of manifestly $I O(2,1)$-covariant field equations proved to be a rather difficult task.

Several approaches have been followed in the literature. We refer to reader to [51] and the introduction of the paper [52] for reviews; see also [29,30,46,47,49]. In the following, we will 
review the results obtained in [53] for the linear relativistic equations whose positive-energy solutions span the massives UIRs where the spin $s$ is neither integer nor half-integer.

The Cortes-Plyushchay equations proposed in [53] read $^{16}$

$$
V_{\mu} \psi=0, \quad V_{\mu}:=s P_{\mu}-i \epsilon_{\mu \nu \lambda} P^{v} \tilde{M}^{\lambda}+m \widetilde{M}_{\mu},
$$

where the three operators $\widetilde{M}_{\mu}:=\frac{1}{2} \epsilon_{\mu \nu \rho} M^{v \rho}$ generate the $\mathfrak{s o}(2,1)$ Lorentz algebra in $D=2+1$ dimensions $\left(i\left[\widetilde{M}_{\mu}, \widetilde{M}_{\nu}\right]=\epsilon_{\mu \nu \rho} \widetilde{M}^{\rho}\right.$ ), so that the quadratic Casimir (4) is equal to $\mathcal{C}_{2}[\mathfrak{s o}(2,1)]=$ $-\widetilde{M}_{\mu} \widetilde{M}^{\mu}$. In the above equations (95), the real number $s$ is assumed to be nonzero. Contracting the above equations with $\widetilde{M}^{\mu}, P^{\mu}$ and $\epsilon^{\mu \nu \lambda} P_{\mu} \widetilde{M}_{\lambda}$ produces the following three equations

$$
\left((s-1) W+m \tilde{M}^{2}\right) \psi=0,\left(s P^{2}+m W\right) \psi=0,\left(P^{2} \widetilde{M}^{2}+W(m-W)\right) \psi=0,
$$

where the scalar $W:=P^{\mu} \widetilde{M}_{\mu}$ is, in three spacetime dimension, the analogue of the PauliLubanski vector. Since by assumption both $s$ and $m$ are non-zero, these three equations are equivalent to

$$
\left(m^{2} \tilde{M}^{2}-s(s-1) P^{2}\right) \psi=0,\left(s P^{2}+m W\right) \psi=0, P^{2}\left(P^{2}+m^{2}\right) \psi=0 .
$$

If one discards the trivial representation of the Poincaré group where $P_{\mu}=0=\widetilde{M}_{\mu}$, one gets the following three equations:

$$
\left(\tilde{M}^{2}+s(s-1)\right) \psi=0, \quad(W-s m) \psi=0, \quad\left(P^{2}+m^{2}\right) \psi=0,
$$

the last two being the Pauli-Lubanski condition and the Klein-Gordon equation, whereas the first sets the quadratic Casimir of the Lorentz group to $\mathcal{C}_{2}[\mathfrak{s o}(2,1)]=s(s-1)$, which indicates that the field $\psi$ takes value in an irrep of the Lorentz group labeled by $s$. The positive-energy solutions of the above field equations (98) transform in the UIR of mass $m$ and spin $s$. More directly, in the Lorentz frame where $p^{\mu}=(m, 0,0)$, the Cortes-Plyushchay equations (95) yield

$$
\left(\widetilde{M}_{0}-s\right) \psi=0, \quad\left(\tilde{M}_{1}-i \widetilde{M}_{2}\right) \psi=0 .
$$

If one takes $L_{ \pm}:=\widetilde{M}_{1} \pm i \widetilde{M}_{2}$ as raising/lowering operators of the Lorentz algebra $\mathfrak{s o}(2,1)$, then these equations assert that the state of momentum $p^{\mu}=(m, 0,0)$ is a lowest-weight state of $\mathfrak{s o}(2,1)$. This implies that the positive-energy solutions are fields taking values in a representation of the Lorentz algebra bounded from below. For $s \notin \frac{1}{2} \mathbb{N}$, one concludes that the field $\psi$ takes values in an infinite-dimensional UIR of the Lorentz algebra $\mathfrak{s o}(2,1)$ belonging to the discrete series.

The cases with $s=-j<0$, where $j \in \frac{1}{2} \mathbb{N}$ is a non-vanishing (half)integer, correspond to the non-unitary spin- $j$ irreducible representations of the Lorentz algebra $\mathfrak{s o}(2,1)$ with quadratic Casimir $\mathcal{C}_{2}[\mathfrak{s o}(2,1)]=j(j+1)$, in which case the Cortes-Plyushchay equation propagates the massive fields with (half)integer spins discussed around (94).

Manifest covariance groups the three components of the equations as the components of a vector, but let us mention that only two of the three equations (95) are enough to produce the third one. These equations are integrable in the sense that the commutator $\left[V_{\mu}, V_{v}\right] \psi$ vanishes on a field $\psi$ solution of (95). We refer to [52] for an extended discussion of these equations.

\section{B.3 Massless representations}

The massless little group in $D=2+1$ spacetime dimensions is $I S O(1) \cong \mathbb{R}$ that is abelian, hence massless UIRs are one-dimensional and labeled by a single real parameter $\mu \in \mathbb{R}$. Therefore, all massless UIRs of the Poincaré group $\operatorname{ISO}(2,1)^{\uparrow}$ have a single physical component. Nevertheless, we will stick to the distinction "helicity" vs "infinite-spin" representations.

\footnotetext{
${ }^{16}$ One can show that the operator $V_{\mu}$ can be obtained by the dimensional reduction of the Siegel-Zwiebach massless operator in (89).
} 


\section{B.3.1 Helicity representations}

The helicity representations correspond to the particular case $\mu=0$. Two case arises whether the representation of the Lorentz group $S O(2,1)^{\uparrow}$ is either single or double valued: the "helicity" is effectively zero or one-half, which corresponds to the fact that a massless field in three spacetime dimensions can always be dualized to a massless scalar or a Dirac spinor, as will be reviewed now. The manifestly covariant field equations are similar to those for the massless helicity cases in $D>3$ studied above, except that only symmetric (spinor-)tensor gauge fields $\varphi_{\mu_{1} \ldots \mu_{s}}=\varphi_{\left(\mu_{1} \ldots \mu_{s}\right)}$ are allowed (the spinor index is not written). Equivalently, only field strengths $\mathcal{K}_{\mu_{1} v_{1}|\ldots| \mu_{s} v_{s}}$ labeled by rectangular two-row Young diagrams are allowed. Moreover, higher (gamma-)traces of those field strengths must be set to zero. Indeed, if in three dimensions one were to set to zero the single (gamma-)trace of the field strength $\mathcal{K}$, one would obtain that the field strength itself should vanish on-shell, resulting in the absence of propagating degrees of freedom. More precisely, upon Hodge-dualizing the $s$ pairs of antisymmetric indices of the spin-s field strength one obtains a totally symmetric (spinor-)tensor

$$
\widetilde{\mathcal{K}}_{\mu_{1} \ldots \mu_{s}}:=\frac{1}{2^{s}} \epsilon_{\mu_{1} v_{1} \rho_{1}} \cdots \epsilon_{\mu_{s} v_{s} \rho_{s}} \mathcal{K}^{v_{1} \rho_{1}|\ldots| v_{s} \rho_{s}},
$$

where the latter (spinor-)tensor is completely symmetric in its spacetime indices.

The closure and coclosure conditions on the field strength $\mathcal{K}$ are equivalent to coclosure and closure condition on its dual:

$$
\partial^{\mu_{1}} \widetilde{\mathcal{K}}_{\mu_{1} \mu_{2} \ldots \mu_{s}}=0, \quad \partial_{\mu} \widetilde{\mathcal{K}}_{v \rho_{1} \ldots \rho_{s-1}}-\partial_{\nu} \widetilde{\mathcal{K}}_{\mu \rho_{1} \ldots \rho_{s-1}}=0 .
$$

The field strength $\widetilde{K}$ begin closed, it is exact:

$$
\widetilde{\mathcal{K}}_{\mu_{1} \ldots \mu_{s}}=p_{\mu_{1}} \ldots p_{\mu_{s}} \phi
$$

where $\phi$ is a (spinor) scalar.

The higher-trace equations on the field strength $\mathcal{K}$ for a propagating, massless helicity representation in three dimensions, are then for bosons

$$
\eta^{\mu_{1} \mu_{2}} \widetilde{\mathcal{K}}_{\mu_{1} \mu_{2} \mu_{3} \ldots \mu_{s}}=0, \quad s>1,
$$

with the usual massless Klein-Gordon and Maxwell equations for $s=0$ and 1, respectively, and for fermions

$$
\gamma^{\mu} \widetilde{\mathcal{K}}_{\mu v_{2} \ldots v_{s}}=0
$$

for the spin $s+\frac{1}{2}>\frac{1}{2}$ cases; the spin- $\frac{1}{2}$ case being of course given by $\gamma^{\mu} \partial_{\mu} \phi=0$, where again, the spinor indices are not written and the three $\gamma^{\mu}$ matrices are three Dirac (in fact Pauli) matrices in $D=2+1$ dimensions.

The conclusion is that all these descriptions of bosonic (respectively, fermionic) massless fields are dual to each others, for all (half-)integer values of the "spin" $s$, in accordance with fact that the positive-energy solutions of the above Bargmann -Wigner equations (102) (respectively, (103), for fermions) carry a single (respectively, double) valued helicity representations of the Poincaré group $\operatorname{ISO}(2,1)^{\uparrow}$. Concretely, these fields are dual a scalar (or spinor) field. More explicitly, the on-shell duality relation between the gauge fields $\varphi_{\mu_{1} \ldots \mu_{s}}$, the field strengths $\mathcal{K}_{\mu_{1} v_{1}|\ldots| \mu_{s} v_{s}}$ and the massless scalar (or spinor) field $\phi$ is (101).

\section{B.3.2 Infinite spin representations}

The positive-energy solutions of the Wigner equations (74)-(76), reviewed in Subsection 5.3.2, transform in the massless infinite-spin representation of the Poincaré group $\operatorname{ISO}(2,1)^{\uparrow}$, labeled by $\mu>0$. The paper [54] provided an extensive discussion of massless infinite-spin particles in $D=2+1$ dimensions. 


\section{B.4 Tachyonic representations}

Finally, in order to be exhaustive, we end this section by mentioning that the relativistic equations (84)-(87) provide an exhaustive solution of the Bargmann-Wigner programme in the tachyonic case. Indeed, the little group $S O(1,1)$ of a spacelike momenta in $D=2+1$ dimensions is Abelian, thus its UIRS of are labeled by a single parameter $s \in \mathbb{R}$.

\section{References}

[1] B. C. Hall, An elementary introduction to groups and representations, (2000), arXiv:math$\mathrm{ph} / 0005032$.

[2] A. Barut and R. Raczka, Theory of group representations and applications, World Scientific, Singapore, ISBN 9789971502164 (1986), doi:10.1142/0352.

[3] X. Bekaert, N. Boulanger, S. de Buyl, F. Dolan and J.-H. Park, Lie algebras: representation and applications, in Proceedings of the First Modave Summer School in Mathematical Physics (2005).

[4] V. Bargmann and E. P. Wigner, Group theoretical discussion of relativistic wave equations, Proc. Natl. Acad. Sci. 34, 211 (1948), doi:10.1073/pnas.34.5.211.

[5] S. Weinberg, The quantum theory of fields, Cambridge University Press, Cambridge, United Kingdom, ISBN 9780521670531 (1995), doi:10.1017/CBO9781139644167.

[6] E. Wigner, On unitary representations of the inhomogeneous Lorentz group, Ann. Math. 40, 149 (1939), doi:10.2307/1968551.

[7] E. P. Wigner, Invariant quantum mechanical equations of motion, in International Atomic Energy Agency, Vienna 59 (1963).

[8] L. Brink, A. M. Khan, P. Ramond and X. Xiong, Continuous spin representations of the Poincaré and super-Poincaré groups, J. Math. Phys. 43, 6279 (2002), doi:10.1063/1.1518138.

[9] W.-K. Tung, Group theory in physics, World Scientific, Singapore, ISBN 9789971966577 (1985), doi:10.1142/0097.

[10] B. Binegar, Relativistic field theories in three dimensions, J. Math. Phys. 23, 1511 (1982), doi:10.1063/1.525524.

[11] M. Hamermesh, Group theory and its applications to physical problems, pp. 544, Dover Reprint Edition (2003).

[12] H. Bacry, Leçons sur la théorie des groupes et les symétries des particules élémentaires, Chapter 4, Dunod (1967).

[13] W. Fulton and J. Harris, Representation theory: a first course, Chapters 4, 6, 15 and 19, Springer (2004).

[14] S. A. Fulling, R. C. King, B. G. Wybourne and C. J. Cummins, Normal forms for tensor polynomials. I. The Riemann tensor, Class. Quantum Grav. 9, 1151 (1992), doi:10.1088/02649381/9/5/003. 
[15] R. C. King, Branching rules for classical Lie groups using tensor and spinor methods, J. Phys. A: Math. Gen. 8, 429 (1975), doi:10.1088/0305-4470/8/4/004.

[16] R. C. King, The dimensions of irreducible tensor representations the orthogonal and symplectic groups, Canad. Jour. Math. 23, 176 (1971), doi:10.4153/CJM-1971-017-2.

[17] M. Dubois-Violette and M. Henneaux, Tensor fields of mixed Young symmetry type and $N$ complexes, Commun. Math. Phys. 226, 393 (2002), doi:10.1007/s002200200610.

[18] E. Inönü and E. P. Wigner, On the contraction of groups and their representations, Proc. Natl. Acad. Sci. 39, 510 (1953), doi:10.1073/pnas.39.6.510.

[19] X. Bekaert and J. Mourad, The continuous spin limit of higher spin field equations, J. High Energ. Phys. 01, 115 (2006), doi:10.1088/1126-6708/2006/01/115.

[20] X. Bekaert and E. D. Skvortsov, Elementary particles with continuous spin, Int. J. Mod. Phys. A 32, 1730019 (2017), doi:10.1142/S0217751X17300198.

[21] I. L. Buchbinder, S. M. Kuzenko, Ideas and methods of supersymmetry and supergravity, or a walk through superspace, Physics Today 49, 64 (1996), doi:10.1063/1.2807514.

[22] W. Siegel, Fields, (1999), arXiv:hep-th/9912205.

[23] X. Bekaert and N. Boulanger, Tensor gauge fields in arbitrary representations of $G L(D, \mathbb{R})$ : Duality and Poincaré lemma, Commun. Math. Phys. 245, 27 (2004), doi:10.1007/s00220-003-0995-1.

[24] X. Bekaert and N. Boulanger, On geometric equations and duality for free higher spins, Phys. Lett. B 561, 183 (2003), doi:10.1016/S0370-2693(03)00409-X.

[25] X. Bekaert and N. Boulanger, Tensor gauge fields in arbitrary representations of $G L(D, R)$. II. Quadratic actions, Commun. Math. Phys. 271, 723 (2007), doi:10.1007/s00220-0060187-x.

[26] I. Bandos, X. Bekaert, J. A. de Azcárraga, D. Sorokin and M. Tsulaia, Dynamics of higher spin fields and tensorial space, J. High Energ. Phys. 05, 031 (2005), doi:10.1088/11266708/2005/05/031.

[27] T. Basile, X. Bekaert and N. Boulanger, Mixed-symmetry fields in de Sitter space: a group theoretical glance, J. High Energ. Phys. 05, 081 (2017), doi:10.1007/JHEP05(2017)081.

[28] W. Siegel and B. Zwiebach, Gauge string fields from the light cone, Nucl. Phys. B 282, 125 (1987), doi:10.1016/0550-3213(87)90679-1.

[29] R. Jackiw and V. P. Nair, Relativistic wave equation for anyons, Phys. Rev. D 43, 1933 (1991), doi:10.1103/PhysRevD.43.1933.

[30] J. L. Cortes and M. S. Plyushchay, Anyons as spinning particles, Int. J. Mod. Phys. A 11, 3331 (1996), doi:10.1142/S0217751X96001590.

[31] M. Fierz, Über die relativistische theorie krafterfreier teilchen mit beliebigem spin, Helv. Phys. Acta XII, 3 (1939).

[32] P. A. M. Dirac, Relativistic wave equations, Proc. R. Soc. Lond. A 155, 447 (1936), doi:10.1098/rspa.1936.0111. 
[33] S. Weinberg, Photons and gravitons in perturbation theory: Derivation of Maxwell's and Einstein's equations, Phys. Rev. 138, B988 (1965), doi:10.1103/PhysRev.138.B988.

[34] C. Fronsdal, Massless fields with integer spin, Phys. Rev. D 18, 3624 (1978), doi:10.1103/PhysRevD.18.3624.

[35] S. Weinberg, Massless particles in higher dimensions, Phys. Rev. D 102, 095022 (2020), doi:10.1103/PhysRevD.102.095022.

[36] E. P. Wigner, Relativistische Wellengleichungen, Z. Physik 124, 665 (1948), doi:10.1007/BF01668901.

[37] A. M. Khan and P. Ramond, Continuous spin representations from group contraction, J. Math. Phys. 46, 053515 (2005), doi:10.1063/1.1897663, [Erratum: ibid. 46, 079901 (2005)].

[38] V. Bargmann, Irreducible unitary representations of the Lorentz group, Ann. Math. 48, 568 (1947), doi:10.2307/1969129.

[39] E. A. Thieleker, The unitary representations of the generalized Lorentz groups, Trans. Amer. Math. Soc. 199, 327 (1974), doi:10.1090/S0002-9947-1974-0379754-8.

[40] V. K. Dobrev, G. Mack, V. B. Petkova, S. G. Petrova, and I. T. Todorov, Harmonic analysis on the n-dimensional Lorentz group and its application to conformal quantum field theory, Springer-Verlag (1977).

[41] I. T. Todorov, M. C. Mintchev, and V. B. Petkova, Conformal invariance in quantum field theory, Scuola normale superiore, Classe di scienze, Chapter IV, Appendix B (1978).

[42] N. Boulanger, C. Iazeolla and P. Sundell, Unfolding mixed-symmetry fields in AdS and the BMV conjecture: I. General formalism, J. High Energ. Phys. 07, 013 (2009), doi:10.1088/1126-6708/2009/07/013.

[43] N. Boulanger, C. Iazeolla and P. Sundell, Unfolding mixed-symmetry fields in AdS and the BMV conjecture. II. Oscillator realization, J. High Energy Phys. 07, 014 (2009), doi:10.1088/1126-6708/2009/07/014.

[44] S. M. Kuzenko and A. E. Pindur, Massless particles in five and higher dimensions, Phys. Lett. B 812, 136020 (2021), doi:10.1016/j.physletb.2020.136020.

[45] A. O. Barut and C. Fronsdal, On non-compact groups. II. Representations of the $2+1$ Lorentz group, Proc. R. Soc. Lond. A 287, 532 (1965), doi:10.1098/rspa.1965.0195.

[46] M. S. Plyushchay, Relativistic particle with torsion, Majorana equation and fractional spin, Phys. Lett. B 262, 71 (1991), doi:10.1016/0370-2693(91)90645-7.

[47] M. S. Plyushchay, The Model of relativistic particle with torsion, Nucl. Phys. B 362, 54 (1991), doi:10.1016/0550-3213(91)90555-C.

[48] I. Tyutin and M. Vasiliev, Lagrangian formulation of irreducible massive fields of arbitrary spin in 2+1 dimensions, (1997), arXiv:hep-th/9704132.

[49] I. V. Gorbunov, S. M. Kuzenko and S. L. Lyakhovich, On the minimal model of anyons, Int. J. Mod. Phys. A 12, 4199 (1997), doi:10.1142/S0217751X97002292. 
[50] N. Boulanger, D. Ponomarev, E. Sezgin and P. Sundell, New unfolded higher spin systems in $\mathrm{AdS}_{3}$, Class. Quantum Grav. 32, 155002 (2015), doi:10.1088/02649381/32/15/155002.

[51] S. Forte, Quantum mechanics and field theory with fractional spin and statistics, Rev. Mod. Phys. 64, 193 (1992), doi:10.1103/RevModPhys.64.193.

[52] P. A. Horváthy, M. S. Plyushchay and M. Valenzuela, Bosons, fermions and anyons in the plane, and supersymmetry, Ann. Phys. 325, 1931 (2010), doi:10.1016/j.aop.2010.02.007.

[53] J. L. Cortés and M. S. Plyushchay, Linear differential equations for a fractional spin field, J. Math. Phys. 35, 6049 (1994), doi:10.1063/1.530727.

[54] P. Schuster and N. Toro, A new class of particle in $2+1$ dimensions, Phys. Lett. B 743, 224 (2015), doi:10.1016/j.physletb.2015.02.050. 